\title{
Exact long time behavior of some regime switching stochastic processes
}

\author{
FILIP LINDSKOG* and ABHISHEK PAL MAJUMDER ${ }^{1 \dagger}$ \\ Department of Mathematics, Stockholm University, SE-106 91 Stockholm, Sweden. \\ E-mail:*lindskog@math.su.se; †abhishek_pal@sutd.edu.sg
}

\begin{abstract}
Regime switching processes have proved to be indispensable in the modeling of various phenomena, allowing model parameters that traditionally were considered to be constant to fluctuate in a Markovian manner in line with empirical findings. We study diffusion processes of Ornstein-Uhlenbeck type where the drift and diffusion coefficients $a$ and $b$ are functions of a Markov process with a stationary distribution $\pi$ on a countable state space. Exact long time behavior is determined for the three regimes corresponding to the expected drift: $E_{\pi} a(\cdot)>0,=0,<0$, respectively. Alongside we provide exact time limit results for integrals of form $\int_{0}^{t} b^{2}\left(X_{S}\right) e^{-2 \int_{s}^{t} a\left(X_{r}\right) d r} d s$ for the three different regimes. Finally, we demonstrate natural applications of the findings in terms of Cox-Ingersoll-Ross diffusion and deterministic SIS epidemic models in Markovian environments. The time asymptotic behaviors are naturally expressed in terms of solutions to the well-studied fixed-point equation in law $X \stackrel{d}{=} A X+B$ with $X \Perp(A, B)$.
\end{abstract}

Keywords: Cox Ingersoll Ross; long time behavior; Ornstein Uhlenbeck; regime switching; SIS epidemic model

\section{Introduction}

Models based on regime switching stochastic processes have received considerable attention for their applications in quantitative finance, actuarial science, economics, biology and ecology. In quantitative finance, volatility, interest rates and asset prices are subjects to risky market environments that fluctuate over different regimes in a Markovian manner. Understanding how critical parameters (that determine stability or instability of the process of interest) characterizing the "switching regimes" vary stochastically over time and affect the long time behavior of the overall process is essential for making short and long term predictions. Examples of such applications are Ang and Timmermann [2], BenSaida [7], Fink et al. [18] and Genon-Catalot et al. [20] in the context of stochastic volatility modelling in financial market; Zhang et al. [42] considering stochastic interest rate models with Markov switching; Hardy [25], Lin et al. [29] and Shen and Kuen Siu [38] studying long term behavior of stock returns and bond pricing. Similar to quantitative finance, regime switching stochastic processes are frequently used in actuarial science for solvency investigations, for example, Abourashchi et al. [1], mortality modeling, for example, Gao et al. [19], and in the context of disability insurance, for example, Djehiche and Löfdahl [15].

Diffusions with Markov switching were initially introduced in Basak et al. [4] as Brownian perturbations of piecewise deterministic models. To the best of our knowledge it is one of the

${ }^{1}$ Corresponding author.

1350-7265 C 2020 ISI/BS 
early references where, under diffusion contexts, stability issues were explored. Monographs containing both the theoretical foundations and applications of regime switching processes are Mao and Yuan [31] and Yin and Zhu [41]. Significant contributions to the theoretical foundation are Shao [35-37]. A common theme of these works is a stochastic dynamical system $\left(Y_{t}, X_{t}\right)_{t \geq 0}$, where the process of interest $Y:=\left(Y_{t}\right)_{t \geq 0}$ is affected by the process $X:=\left(X_{t}\right)_{t \geq 0}$ that describes the dynamics of a switching environment. For a class of general diffusion processes $Y$ the aforementioned works investigated necessary and sufficient conditions under which properties related to stability/instability such as geometric/polynomial ergodicity in Shao [35,36], positive/null recurrence or transience in Shao [37], explosivity, existence and uniqueness of moments of stationary distributions hold. In a similar context Benaïm and Lobry [6] (and references therein) addresses questions related with survival or extinction of competing species in a Lotka-Volterra model influenced by switching parameters in terms of the underlying hidden Markov environment. A main theme is the analysis of persistence (see Section 4 or Theorem 4.1 in [6]) phrased in terms of so-called Lyapunov drift type criteria and similar concepts. In Cloez and Hairer [12], a large class of general regime switching Markov processes are considered where a type of condition referred to as geometric contractivity ensures exponential stability of the overall process. In contrast to the general stability results described above, there are very few works giving exact characterizations of long time behaviors, which are inevitably model specific. In this paper, we analyze the long time behavior of processes of Ornstein-Uhlenbeck type and Cox-IngersollRoss models in a regime switching context, and provide exact explicit characterizations. To our knowledge, such explicit characterizations have not appeared in the literature.

The initial object of study in this paper is an $\mathbb{R}$-valued Ornstein-Uhlenbeck process in a Markovian environment, denoted by $Y=\left(Y_{t}\right)_{t \geq 0}$, defined as the solution to the SDE

$$
d Y_{t}=-a\left(X_{t}\right) Y_{t} d t+b\left(X_{t}\right) d W_{t}, \quad Y_{0}=y_{0} \in \mathbb{R},
$$

where $\left(W_{t}\right)_{t \geq 0}$ is standard Brownian motion which is independent of $X:=\left(X_{t}\right)_{t \geq 0}$ that represents the background environment. $X$ is an $S$-valued, where $S$ is a countable set, jump type process with jump intensities $\lambda_{i j} \in \mathbb{R}_{+}$, for $(i, j) \in S^{2}$ with $i \neq j$, satisfying

$$
P\left[X_{t+\delta}=j \mid X_{t}=i, Y_{t}=x\right]= \begin{cases}\lambda_{i j} \delta+o(\delta), & \text { if } i \neq j, \\ 1+\lambda_{i i} \delta+o(\delta), & \text { if } i=j,\end{cases}
$$

with notation $\lambda_{i i}:=-\sum_{j \neq i \in S} \lambda_{i j}$. (A more general setting where state dependent intensities $\lambda_{i j}(x)$ were considered is found in Shao [36] and Cloez and Hairer [12], investigating joint ergodicity of $\left(X_{t}, Y_{t}\right)_{t \geq 0}$. $)$ The process $X$ is a continuous-time Markov chain, with respect to its natural filtration $\left(\mathcal{F}_{t}^{X}\right)_{t \geq 0}$, satisfying the hidden Markovian assumption:

$$
X_{t} \Perp_{X_{s}} Y_{s} \text { for all } t>s,
$$

saying that for all $t>s, X_{t}$ is conditionally independent of $Y_{s}$ given $X_{s}$.

The functions $a, b: S \rightarrow \mathbb{R}$ are arbitrary (as long as a pathwise unique weak solution of (1.1) can be insured) denoting the drift and the diffusion functions, respectively. $\left(X_{t}, Y_{t}\right)_{t \geq 0}$ is a Markov process with respect to its natural filtration $\left(\mathcal{F}_{t}^{X, Y}\right)_{t \geq 0}$.

We say that a stochastic process $X:=\left(X_{t}\right)_{t \geq 0}$ is ergodic if there exists a probability distribution $\mu$ such that, regardless of $X_{0}$, the distribution of $X_{t}$ converges weakly to the limiting 
distribution $\mu$ as $t \rightarrow \infty$. If $X$ is an irreducible and ergodic Markov chain in a countable state space $S$, then the limiting distribution $\mu$ is its unique stationary distribution (or invariant distribution), see, for example, Norris [33].

Throughout the paper, we assume that the hidden Markov chain $X$ is ergodic with stationary distribution $\pi:=\left\{\pi_{j}: j \in S\right\}$. The process $Y$ is attractive or stable if $E_{\pi} a(\cdot)>0$ holds, otherwise it is divergent if $E_{\pi} a(\cdot)<0$ and null recurrent if $E_{\pi} a(\cdot)=0$ (which can be shown using the Wasserstein contraction ideas from Hairer [24] or Lyapunov function based constructions in Shao [37]). Under the stability assumption $E_{\pi} a(\cdot)>0$, a trichotomy of possible tail behaviors of the stationary distribution was established in Bardet et al. [3]. Using Fourier analysis techniques, Zhang and Wang [43] provided precise results on ergodicity when $|S|=2$. A key contribution of our paper is a precise result on ergodicity including an explicit representation for the stationary distribution when $S$ is a countable state space, allowing computation of probabilities $\lim _{t \rightarrow \infty} P\left[Y_{t}>y\right]$. The result is generalized by generalizing the model (1.1) in different ways. Under the instability assumption $E_{\pi} a(\cdot) \leq 0$ no stationary distribution exists and we determine how $Y$ diverges by providing weak limits for the scaled fluctuations $\left(\log \left|Y_{t}\right|\right) / \sqrt{t}$ which translate to the behavior of $\left|Y_{t}\right|^{1 / \sqrt{t}}$. In all long time results, we describe how introducing a regime switching component leads to mixture type representations characterizing the long time behavior.

In parallel to the characterization of the long time behavior of the model (1.1), we provide the corresponding explicit characterization of the long time behavior of integrals of the type

$$
\int_{0}^{t} d\left(X_{S}\right) e^{-\int_{s}^{t} c\left(X_{r}\right) d r} d s
$$

for the three regimes $E_{\pi} c(\cdot)>0,=0,<0$ corresponding to positive recurrence, null recurrence and transience.

Several previous works, for example, Gjessing and Paulsen [21], Bertoin and Yor [8], Maulik and Zwart [32], Behme and Lindner [5], Zhang et al. [42] and Feng et al. [17] have studied exponential functionals of Lévy processes. For instance, in Gjessing and Paulsen [21] the asymptotic behavior of integrals of the form $\int_{0}^{t} e^{-R_{s}} d P_{s}$ as $t \rightarrow \infty$ was explored, where $P$ and $R$ are independent Lévy processes and the property

$$
\left(P_{t}, R_{t}\right) \stackrel{d}{=}\left(P_{s}, R_{s}\right)+\left(\widetilde{P}_{t-s}, \widetilde{R}_{t-s}\right), \quad(\tilde{P}, \tilde{R}) \text { is an independent copy of }(P, R),
$$

helps the analysis significantly. We derive exact limit results for integrals of the type (1.4), where $X$ is a continuous time Markov chain on a countable state space. The asymptotic analysis requires quite different methods from those used for the corresponding analysis for exponential functionals of Lévy processes. Asymptotic analysis similar to the one presented in the current paper was done in Bardet et al. [3]. Proposition 4.1 in [3] yields asymptotic bounds for (1.4), but not the exact asymptotic behavior that we present here.

The paper is organized as follows. Section 2 sets notation and presents basic model assumptions. In Section 3, exact long time characterizations for the stochastic process (1.1), and different generalizations, are presented under assumptions corresponding to the stable regime of the aforementioned process (1.1). Section 4 presents long time characterizations corresponding to the unstable regime when no stationary distribution exists. Section 5 contains applications of 
the findings in Sections 3 and 4 to the CIR model, originally introduced as a model for interest rates, and to SIS models used in epidemiology. The proofs are found in Section 6 and in the supplementary material [30].

\section{Preliminaries and model assumptions}

Whenever relevant, random elements appearing are assumed to be defined on a common probability space with probability measure $P$ and expectation operator $E$. $\mathbb{R}^{d}$ denotes the $d$ dimensional Euclidean space with the usual Euclidean norm $|\cdot|$. The set of natural numbers is denoted by $\mathbb{N}$. Cardinality of a finite set $S$ is denoted by $|S|$. For any given sequence $\left(a_{n}\right)_{n \geq 1}$, define $\left(a_{n}^{\max }\right)_{n \geq 1}$ as the sequence of running maxima $a_{n}^{\max }:=\max _{1 \leq k \leq n} a_{k}$.

For a Polish space $S$, let $\mathcal{B}(S)$ be its Borel $\sigma$-field and let $\mathcal{P}(S)$ denote the class of probability measures on $S . \mathcal{P}(S)$ is equipped with the topology of weak convergence. For $x \in S, \delta_{x} \in \mathcal{P}(S)$ denotes the Dirac measure that puts unit mass at $x$. The probability distribution of an $S$-valued random variable $X$ will be denoted as $\mathcal{L}(X) . X \sim \mu$ means that $\mu \in \mathcal{P}(S)$ and $\mu=\mathcal{L}(X)$. Convergence in distribution of an $S$-valued sequence $\left(X_{n}\right)_{n \geq 1}$ to an $S$-valued random variable $X$ will be written as $X_{n} \stackrel{d}{\rightarrow} X$, or $\mathcal{L}\left(X_{n}\right) \stackrel{w}{\rightarrow} \mathcal{L}(X)$, where $w$ stands for weak convergence. We write $X_{n} \stackrel{\text { a.s. }}{\rightarrow} X$ and $X_{n} \stackrel{P}{\rightarrow} X$ for convergence almost surely and in probability, respectively.

The transition kernels of a Markov process are defined as the maps $P_{s, t}:(S, \mathcal{B}(S)) \rightarrow[0,1]$ such that for all $t \geq s \geq 0, P_{s, t}(\cdot, A)$ is $\mathcal{B}(S)$-measurable for each $A \in \mathcal{B}(S)$ and $P_{s, t}(i, \cdot) \in \mathcal{P}(S)$ for each $i \in S$. The distribution of the Markov process is determined by the transition kernels $P_{s, t}$ together with the initial distribution $v_{0}$. The marginal distribution of the Markov process at time $t$ is $v_{0} P_{0, t}(\cdot)=\int_{S} P_{0, t}(x, \cdot) v_{0}(d x)$. We will consider only time-homogeneous Markov processes corresponding to transition kernels satisfying $P_{s, t}=P_{0, t-s}$ and use the notation $P_{t}:=P_{0, t}$. $P_{t} f(\cdot)$ is the corresponding transition operator given by $P_{t} f(x)=\int_{S} f(y) P_{t}(x, d y)$ for functions $f:(S, \mathcal{B}(S)) \rightarrow(\mathbb{R}, \mathcal{B}(\mathbb{R}))$. A time-homogeneous Markov process in a countable state space $S$ is irreducible if for any $i, j \in S, P_{t}(i, j):=P_{t}(i,\{j\})>0$ for some $t>0$. A timehomogeneous Markov process with transition kernels $P_{t}$ has a stationary distribution (or invariant distribution) $\mu \in \mathcal{P}(S)$ if $\mu P_{t}=\mu$ holds for all $t>0$. A continuous-time Markov chain is a pure jump-type time-homogeneous Markov process on a countable state space. An irreducible and non-explosive continuous-time Markov chain having a stationary distribution is ergodic (Theorem 3.6.2 in Norris [33]).

Consider a probability measure $\pi$ on a countable set $S$ such that $\pi(A)=\sum_{i \in A} \pi_{i}$ for any $A \in \mathcal{B}(S)$ and a set of probability measures $\left\{\mu_{j}: j \in S\right\}$. Then

$$
\sum_{j \in S} \delta_{U}(\{j\}) Z_{j}, \quad U \Perp\left(Z_{j}\right)_{j \in S}, U \sim \pi, Z_{j} \sim \mu_{j},
$$

is a random variable whose distribution is the mixture distribution $\sum_{j \in S} \pi_{j} \mu_{j}$. For a given bivariate random variable $(A, B)$, the following time series is referred to as a stochastic recurrence equation (in short SRE, also referred to as random coefficient AR(1))

$$
Z_{n+1}=A_{n+1} Z_{n}+B_{n+1} \quad \text { with }\left(A_{i}, B_{i}\right) \stackrel{\text { i.i.d }}{\sim} \mathcal{L}(A, B), Z_{n} \Perp\left(A_{n+1}, B_{n+1}\right)
$$


for an arbitrary initial value $Z_{0}=z_{0} \in \mathbb{R}$. Let $\log ^{+}|a|:=\log (\max (|a|, 1))$. If

$$
P[A=0]=0, \quad E \log |A|<0 \quad \text { and } \quad E \log ^{+}|B|<\infty,
$$

then $\left(Z_{n}\right)$ has a unique causal ergodic strictly stationary solution solving the following fixedpoint equation in law:

$$
Z \stackrel{d}{=} A Z+B \quad \text { with } Z \Perp(A, B) .
$$

The condition $P[A x+B=x]<1$, for all $x \in \mathbb{R}$, rules out degenerate solutions $Z=x$ a.s. We refer to Corollary 2.1.2 and Theorem 2.1.3 in Buraczewski et al. [9] for further details.

We denote by $\mathrm{N}\left(\mu, \sigma^{2}\right)$ and $\operatorname{Exp}(\lambda)$, respectively, the normal distribution with mean $\mu$ and variance $\sigma^{2}$ and the exponential distribution with mean $1 / \lambda$.

Throughout the rest of this paper we will assume the following.

Assumption 1. $S$ is a countable set and $X:=\left(X_{t}\right)_{t \geq 0}$ is an irreducible and ergodic continuoustime Markov chain in $S$ with stationary distribution $\pi:=\left\{\pi_{j}: j \in S\right\}$. X satisfies (1.2).

It follows from Assumption 1 that $X$ is positive recurrent and $\pi_{j}>0$ for all $j \in S$. Fix a state $j \in S$. Let $\tau_{0}^{j}$ be the first time $X$ visits state $j$ and let $T_{0}^{j}$ be the amount of time $X$ stays there before leaving state $j$. Define recursively, for $k \geq 1$,

$$
\tau_{k}^{j}:=\inf \left\{t>\tau_{k-1}^{j}+T_{k-1}^{j}: X_{t}=j\right\}, \quad T_{k}^{j}:=\inf \left\{t>\tau_{k}^{j}: X_{t} \neq j\right\}-\tau_{k}^{j} .
$$

After (re)visiting state $j$ at time $\tau_{k}^{j}, X$ stays there for an amount of time denoted by $T_{k}^{j}$. The sequence $\left(T_{k}^{j}\right)_{k \geq 0}$ is an i.i.d. sequence with $\operatorname{Exp}\left(-\lambda_{j j}\right)$-distributed terms. Define $I_{k}^{j}:=\left[\tau_{k-1}^{j}, \tau_{k}^{j}\right)$ and notice that the renewal cycle lengths $\left|I_{k}^{j}\right|:=\tau_{k}^{j}-\tau_{k-1}^{j}$ form an i.i.d. sequence $\left(\left|I_{k}^{j}\right|\right)_{k \geq 1}$. A consequence of positive recurrence of $X$ is that $E\left|I_{k}^{j}\right|<\infty$ for any $(j, k) \in S \times \mathbb{N}$. Let

$$
g_{t}^{j}:=\max \left(\sup \left\{n \in \mathbb{N}: \tau_{n}^{j} \leq t\right\}, 0\right), \quad \sup \varnothing:=-\infty,
$$

that is, the number of times the chain $X$ revisits the state $j$ before time $t$. Positive recurrence of $X$ implies that $g_{t}^{j} \stackrel{\text { a.s. }}{\rightarrow} \infty$ as $t \rightarrow \infty$.

\section{The stable regime}

In this section, we study long time behavior of the joint process $(Y, X):=\left(Y_{t}, X_{t}\right)_{t \geq 0}$ and processes defined in terms of certain functionals of $\left(X_{t}\right)_{t \geq 0}$ under conditions ensuring that convergence in distribution holds as $t \rightarrow \infty$. Together with Assumption 1, the following assumption ensures the existence of a stationary distribution for $(Y, X)$.

Assumption 2. The $S$-valued process $X$ and the functions $a, b: S \rightarrow \mathbb{R}$ satisfy

(a) $a$ is integrable with respect to $\pi$, and $E_{\pi} a(\cdot)>0$. 
(b) For every $j \in S, E\left[\log ^{+} \int_{\tau_{0}^{j}}^{\tau_{1}^{j}} b^{2}\left(X_{S}\right) e^{-2 \int_{s}^{\tau_{1}^{j}} a\left(X_{r}\right) d r} d s\right]<\infty$.

Remark 3.1. Assumption 2 corresponds, in the current setting, to the general condition (2.1) for existence of a stationary solution to the stochastic recurrence equation

$$
Z_{j, n+1}=e^{-2 \int_{\tau_{n}^{j}}^{\tau_{n+1}^{j}} a\left(X_{s}\right) d s} Z_{j, n}+\int_{\tau_{n}^{j}}^{\tau_{n+1}^{j}} b^{2}\left(X_{s}\right) e^{-2 \int_{s}^{\tau_{n+1}^{j}} a\left(X_{r}\right) d r} d s
$$

with affine invariant solution of the form

$$
Z_{j} \stackrel{d}{=} e^{-2 \int_{\tau_{0}^{j}}^{\tau_{1}^{j}} a\left(X_{s}\right) d s} Z_{j}+\int_{\tau_{0}^{j}}^{\tau_{1}^{j}} b^{2}\left(X_{S}\right) e^{-2 \int_{s}^{\tau_{1}^{j}} a\left(X_{r}\right) d r} d s
$$

If Assumption 2(a) holds but not Assumption 2(b), then results similar to Theorem 1.1 in $\mathrm{Bu}$ raczewski and Iksanov [10] hold. Notice that if $\sup _{j \in S}|a(j)|<\infty$ and $E_{\pi} b^{2}(\cdot)<\infty$, then Assumption 2(b) follows immediately from Assumption 1 as a consequence of the inequalities $\log ^{+}|a b| \leq \log ^{+}|a|+\log ^{+}|b|$ and $\log ^{+}|a| \leq|a|$ for any $a, b$.

An explicit expression for the stationary distribution of the joint process $(Y, X)$ is the following.

Theorem 1. Under Assumptions 1 and 2, the stationary distribution of the joint process $(Y, X)$ can be expressed as a scale mixture of Gaussians as follows:

$$
\left(Y_{t}, X_{t}\right) \stackrel{d}{\rightarrow}\left(\sum_{j \in S} \delta_{U}(\{j\}) Z_{j}, U\right) \quad \text { as } t \rightarrow \infty
$$

where $U \Perp\left(Z_{j}\right)_{j \in S}, U \sim \pi, Z_{j} \stackrel{d}{=} \sqrt{V_{j}} N, V_{j} \Perp N, N \sim \mathrm{N}(0,1)$ and

$$
V_{j} \stackrel{d}{=} b^{2}(j) \int_{0}^{T^{j}} e^{-2 a(j)\left(T^{j}-s\right)} d s+e^{-2 a(j) T^{j}} V_{j}^{*},
$$

where $T^{j} \sim \operatorname{Exp}\left(-\lambda_{j j}\right)$ is independent of $V_{j}^{*}$, and $\mathcal{L}\left(V_{j}^{*}\right)$ is the unique solution to (2.2) with $(A, B)$ having the distribution of

$$
\left(e^{-2 \int_{\tau_{0}^{j}}^{\tau_{1}^{j}} a\left(X_{s}\right) d s}, \int_{\tau_{0}^{j}}^{\tau_{1}^{j}} b^{2}\left(X_{s}\right) e^{-2 \int_{s}^{\tau_{1}^{j}} a\left(X_{r}\right) d r} d s\right)
$$

The proof of Theorem 1 is found in Section 6.1. 
Remark 3.2. Theorem 1 may be generalized by instead of the model (1.1) considering the more general Ornstein-Uhlenbeck model

$$
d Y_{t}=\left(c\left(X_{t}\right)-a\left(X_{t}\right) Y_{t}\right) d t+b\left(X_{t}\right) d W_{t}, \quad Y_{0}=y \in \mathbb{R},
$$

for an arbitrary function $c: S \rightarrow \mathbb{R}$. The stationary distribution of $(Y, X)$ can be determined under the assumptions of Theorem 1 and the additional assumption (ensured by $E_{\pi}|c(\cdot)|<\infty$ ):

$$
E \log ^{+} \int_{\tau_{0}^{j}}^{\tau_{1}^{j}}\left|c\left(X_{S}\right)\right| e^{-2 \int_{s}^{\tau_{1}^{j}} a\left(X_{r}\right) d r} d s<\infty \quad \text { for all } j \in S .
$$

The stationary distribution is given by

$$
\left(Y_{t}, X_{t}\right) \stackrel{d}{\rightarrow}\left(\sum_{j \in S} \delta_{U}(\{j\}) Z_{j}, U\right) \quad \text { as } t \rightarrow \infty
$$

where $U \Perp\left(Z_{j}\right)_{j \in S}, U \sim \pi, Z_{j} \stackrel{d}{=} M_{j}+\sqrt{V_{j}} N,\left(M_{j}, V_{j}\right) \Perp N, N \sim \mathrm{N}(0,1)$ and

$$
\left(\begin{array}{c}
M_{j} \\
V_{j}
\end{array}\right) \stackrel{d}{=}\left(\begin{array}{c}
c(j) \int_{0}^{T^{j}} e^{-a(j)\left(T^{j}-s\right)} d s+e^{-a(j) T^{j}} M_{j}^{*} \\
b^{2}(j) \int_{0}^{T^{j}} e^{-2 a(j)\left(T^{j}-s\right)} d s+e^{-2 a(j) T^{j}} V_{j}^{*}
\end{array}\right),
$$

where $T^{j} \sim \operatorname{Exp}\left(-\lambda_{j j}\right)$ is independent of $\left(M_{j}^{*}, V_{j}^{*}\right)$ and $\mathcal{L}\left(M_{j}^{*}, V_{j}^{*}\right)$ is the unique solution to

$$
\left(\begin{array}{c}
M_{j}^{*} \\
V_{j}^{*}
\end{array}\right) \stackrel{d}{=}\left[\begin{array}{cc}
\sqrt{A_{j}} & 0 \\
0 & A_{j}
\end{array}\right]\left(\begin{array}{c}
M_{j}^{*} \\
V_{j}^{*}
\end{array}\right)+\left(\begin{array}{c}
C_{j} \\
B_{j}
\end{array}\right),\left(M_{j}^{*}, V_{j}^{*}\right) \Perp\left(A_{j}, B_{j}, C_{j}\right),
$$

with $\left(A_{j}, B_{j}, C_{j}\right)$ having the distribution of

$$
\left(e^{-\int_{\tau_{0}^{j}}^{\tau_{1}^{j}} 2 a\left(X_{s}\right) d s}, \int_{\tau_{0}^{j}}^{\tau_{1}^{j}} b^{2}\left(X_{S}\right) e^{-2 \int_{s}^{\tau_{1}^{j}} a\left(X_{r}\right) d r} d s, \int_{\tau_{0}^{j}}^{\tau_{1}^{j}} c\left(X_{S}\right) e^{-\int_{s}^{\tau_{1}^{j}} a\left(X_{r}\right) d r} d s\right)
$$

with notations used in Theorem 1. This generalization of Theorem 1 follows from expressing $Y$ in (3.2) as

$$
Y_{t}=Y_{0} e^{-\int_{0}^{t} a\left(X_{r}\right) d r}+\int_{0}^{t} c\left(X_{S}\right)\left(e^{-\int_{s}^{t} a\left(X_{r}\right) d r}\right) d s+\int_{0}^{t} b\left(X_{S}\right) e^{-\int_{s}^{t} a\left(X_{r}\right) d r} d W_{s} .
$$

The characterization (3.3) follows along the lines of the proof of Theorem 1 by modifying the proof of Lemma 6.1 by determining the weak limit as $t \rightarrow \infty$ of

$$
\left(e^{-\int_{0}^{t} a\left(X_{r}\right) d r}, \int_{0}^{t} c\left(X_{S}\right) e^{-\int_{s}^{t} a\left(X_{r}\right) d r} d s, \int_{0}^{t} b^{2}\left(X_{S}\right) e^{-2 \int_{s}^{t} a\left(X_{r}\right) d r} d s\right) .
$$


Remark 3.3. Theorem 1 can be extended further by replacing the standard Brownian motion $W$ by an arbitrary Lévy process $L$ in (1.1):

$$
d Y_{t}=-a\left(X_{t}\right) Y_{t} d t+b\left(X_{t}\right) d L_{t}, \quad Y_{0}=y \in \mathbb{R} .
$$

Exact long time behavior can be determined from the expression

$$
Y_{t}=Y_{0} e^{-\int_{0}^{t} a\left(X_{r}\right) d r}+\int_{0}^{t} b\left(X_{s}\right) e^{-\int_{s}^{t} a\left(X_{r}\right) d r} d L_{s}
$$

under the same assumptions as in Theorem 1 except that Assumption 2(b) is replaced by

$$
E \log ^{+}\left|\int_{\tau_{0}^{j}}^{\tau_{1}^{j}} b\left(X_{s}\right) e^{-\int_{s}^{\tau_{1}^{j}} a\left(X_{r}\right) d r} d L_{S}\right|<\infty \quad \text { for all } j \in S
$$

The stationary distribution can be expressed as

$$
\left(Y_{t}, X_{t}\right) \stackrel{d}{\rightarrow}\left(\sum_{j \in S} \delta_{U}(\{j\}) Z_{j}, U\right) \quad \text { as } t \rightarrow \infty
$$

where $U \Perp\left(Z_{j}\right)_{j \in S}$ and

$$
Z_{j} \stackrel{d}{=} b(j) \int_{0}^{T^{j}} e^{-a(j)\left(T^{j}-s\right)} d L_{s}+e^{-a(j) T^{j}} Z_{j}^{*}
$$

where $T^{j} \sim \operatorname{Exp}\left(-\lambda_{j j}\right), L$ and $Z_{j}^{*}$ are independent, and $\mathcal{L}\left(Z_{j}^{*}\right)$ is the unique solution to (2.2) with $(A, B)$ having the distribution of

$$
\left(e^{-\int_{\tau_{0}^{j}}^{\tau_{1}^{j}} a\left(X_{s}\right) d s}, \int_{\tau_{0}^{j}}^{\tau_{1}^{j}} b\left(X_{S}\right) e^{-\int_{s}^{\tau_{1}^{j}} a\left(X_{r}\right) d r} d L_{s}\right)
$$

More details are found in the supplementary material [30]. The special case $L=W$ corresponds to $Z_{j} \stackrel{d}{=} \sqrt{V_{j}} N$ with $\left(V_{j}, N\right)$ as in Theorem 1 .

Remark 3.4. Theorem 1 together with Mill's ratio inequalities yield tail bounds for the stationary distribution $\mathcal{L}\left(Y_{\infty}\right)$, writing $Y_{t} \stackrel{d}{\rightarrow} Y_{\infty}$ as $t \rightarrow \infty$ for the marginal convergence in (3.1). With $\mu_{j}:=\mathcal{L}\left(V_{j}\right)$,

$$
\sum_{j \in S} \pi_{j} \int_{\mathbb{R}^{+}} \frac{t \phi(t / \sqrt{\sigma})}{1+t^{2}} \mu_{j}(d \sigma) \leq P\left[Y_{\infty}>t\right] \leq \sum_{j \in S} \pi_{j} \int_{\mathbb{R}^{+}} \frac{\phi(t / \sqrt{\sigma})}{t} \mu_{j}(d \sigma),
$$

where $\phi$ is the density function of the standard normal distribution. 
Remark 3.5. Moments for the stationary distribution of $Y$ in (3.5) can be computed recursively using the representation for $\left(A_{j}, B_{j}\right)$ in (3.6). From $Z_{j}^{*} \stackrel{d}{=} A_{j} Z_{j}^{*}+B_{j}$ follows that, for $m \in \mathbb{N}$,

$$
\left(Z_{j}^{*}\right)^{m}\left(1-A_{j}^{m}\right)=\sum_{k=0}^{m-1}\left(\begin{array}{c}
m \\
k
\end{array}\right) A_{j}^{k} B_{j}^{m-k}\left(Z_{j}^{*}\right)^{k} .
$$

If there exists $n \in \mathbb{N}$ such that $E A_{j}^{m}<\infty$ and $E A_{j}^{k} B_{j}^{m-k}<\infty$ for $0 \leq k \leq m \leq n$, then $E\left|Z_{j}^{*}\right|^{n}<$ $\infty$ and independence between $Z_{j}^{*}$ and $\left(A_{j}, B_{j}\right)$ gives following recursive relation of moments

$$
E\left[\left(Z_{j}^{*}\right)^{m}\right]=\frac{1}{1-E A_{j}^{m}} \sum_{k=0}^{m-1}\left(\begin{array}{c}
m \\
k
\end{array}\right) E\left[A_{j}^{k} B_{j}^{m-k}\right] E\left[\left(Z_{j}^{*}\right)^{k}\right] .
$$

From the above representations moments of the limit distribution

$$
E\left[Y_{\infty}^{m}\right]=\sum_{j \in S} \pi_{j} E\left[\left(b(j) \int_{0}^{T^{j}} e^{-a(j)\left(T^{j}-s\right)} d L_{s}+e^{-a(j) T^{j}} Z_{j}^{*}\right)^{m}\right]
$$

can be computed using the independence of $T^{j}, L, Z_{j}^{*}$.

Remark 3.6. Theorem 1 can be generalized by allowing $Y$ to be a vector valued OrnsteinUhlenbeck process. In that case, when both drift and diffusion functions $a, b$ are matrix valued functions of the hidden Markov process $X$, stability conditions will change in a nontrivial way which require careful analysis. Theorem 1 and the methodology used for proving the theorem can be extended to general regime switching dynamics that is marginal of a Markov renewal process (also known as semi-Markov process), where for every regime $j \in S$ the regime process spends a random time before moving to another regime such that the time it stays there has finite mean but is not exponentially distributed. Allowing for an infinite mean would make the analysis substantially more complicated. Since a semi-Markov process is in general non-Markovian, instead of a stationary distribution one should use the similar notion of a limiting distribution for investigating exact long time behavior.

In many applications integrals of the form

$$
F_{t}:=\int_{0}^{t} d\left(X_{S}\right) e^{-\int_{s}^{t} c\left(X_{r}\right) d r} d s
$$

appear for functions $c, d: S \rightarrow \mathbb{R}$ and $X$ being a regime process satisfying Assumption 1 . The following proposition addresses the long time behavior of $F_{t}$ under the stability regime $E_{\pi} c(\cdot)>$ 0 and a suitable integrability property of $d$ in the form of the following assumption.

Assumption 3. The $S$-valued process $X$ and the functions $c, d: S \rightarrow \mathbb{R}$ satisfy

(a) $c$ is integrable with respect to $\pi$, and $E_{\pi} c(\cdot)>0$. 
(b) For every $j \in S, E\left[\log ^{+}\left|\int_{\tau_{0}^{j}}^{\tau_{1}^{j}} d\left(X_{S}\right) e^{-\int_{s}^{\tau_{1}^{j}} c\left(X_{r}\right) d r} d s\right|\right]<\infty$.

The difference between Assumption 2 and Assumption 3 is that in the latter the function $d$ can take negative values in contrast to only positive values for $b^{2}$ appearing in Assumption 2.

Proposition 3.7. Under Assumptions 1 and $3, F_{t}$ in (3.7) satisfies

$$
F_{t} \stackrel{d}{\rightarrow} \sum_{j \in S} \delta_{U}(\{j\}) V_{j} \quad \text { as } t \rightarrow \infty
$$

where $U \sim \pi, U \Perp\left(V_{j}\right)_{j \in S}$ and

$$
V_{j} \stackrel{d}{=} d(j) \int_{0}^{T^{j}} e^{-c(j)\left(T^{j}-s\right)} d s+e^{-c(j) T_{j}} V_{j}^{*},
$$

where $T^{j} \sim \operatorname{Exp}\left(-\lambda_{j j}\right)$ is independent of $V_{j}^{*}$, and $\mathcal{L}\left(V_{j}^{*}\right)$ is the unique solution to (2.2) with $(A, B)$ having the distribution of

$$
\left(e^{-\int_{\tau_{0}^{j}}^{\tau_{1}^{j}} c\left(X_{s}\right) d s}, \int_{\tau_{0}^{j}}^{\tau_{1}^{j}} d\left(X_{S}\right) e^{-\int_{s}^{\tau_{1}^{j}} c\left(X_{r}\right) d r} d s\right)
$$

The proof of Proposition 3.7 is found in the supplementary material [30].

Remark 3.8. The Goldie-Kesten theorem (Theorem 2.4.4 in Buraczewski et al. [9]) characterizes heavy-tailed behavior of the solution to the fixed-point equation (2.2). If $A \geq 0$ a.s. and $\mathcal{L}(\log A \mid A>0)$ is non-arithmetic, $P[A x+B=x]<1$ for all $x \in \mathbb{R}$, and there exists $v>0$ such that

$$
E A^{v}=1, \quad E|B|^{v}<\infty, \quad E A^{v} \log ^{+} A<\infty,
$$

then there exist constants $c_{+}, c_{-} \geq 0$ with $c_{+}+c_{-}>0$ such that

$$
P[X>x] \sim c_{+} x^{-v}, \quad P[X<-x] \sim c_{-} x^{-v} \text { as } x \rightarrow \infty .
$$

This result is applicable to the stationary distribution of $Y$ in Theorem 1 if $\inf _{j \in S} a(j)<0$. Let

$$
v_{j}:=\sup \left\{c>0: E e^{-c \int_{\tau_{0}^{j}}^{\tau_{1}^{j}} a\left(X_{s}\right) d s}<1\right\}, \quad v^{*}:=\inf _{j \in S} v_{j} .
$$

If

$$
\sup _{j \in S} E\left[\left(\int_{\tau_{0}^{j}}^{\tau_{1}^{j}} b^{2}\left(X_{s}\right) e^{-2 \int_{s}^{\tau_{1}^{j}} a\left(X_{r}\right) d r} d s\right)^{v^{*} / 2}\right]<\infty
$$


and

$$
\sup _{j \in S} E\left[e^{-v^{*} \int_{\tau_{0}^{j}}^{\tau_{1}^{j}} a\left(X_{s}\right) d s} \log ^{+} e^{-2 \int_{\tau_{0}^{j}}^{\tau_{1}^{j}} a\left(X_{s}\right) d s}\right]<\infty,
$$

then the left and right tails of the symmetric distribution of

$$
\sum_{j \in S} \delta_{U}(\{j\}) \sqrt{V_{j}} N
$$

are regularly varying with index $v^{*}$. The statement follows since $V_{j}$ is a stochastic affine transformation of $V_{j}^{*}$ by random variables having finite moments of all orders, and since the standard normal distribution has finite moments of all orders. The indices $v_{j}$ can be estimated from the sample paths of $X$ through the empirical estimator

$$
\widehat{v}_{n, j}:=\inf \left\{c>0: \frac{1}{n} \sum_{i=1}^{n} e^{-c \int_{I_{i}^{j}} a\left(X_{s}\right) d s}=1\right\}, \quad \inf \varnothing:=+\infty,
$$

of $v_{j}$ based on $n$ regenerating intervals $\left(I_{i}^{j}\right)_{i=1}^{n}$. Therefore, $v^{*}$ may be estimated iteratively as the limit of

$$
\widehat{v}_{n, j_{k+1}}^{*}:=\min \left(\widehat{v}_{n, j_{k}}^{*}, \inf \left\{c \in\left(0, \widehat{v}_{n, j_{k}}^{*}\right): \frac{1}{n} \sum_{i=1}^{n} e^{-c \int_{I_{i}^{j}} a\left(X_{s}\right) d s}=1\right\}\right), \quad k=1, \ldots,|S| .
$$

Another representation for the tail index $\inf _{j \in S} v_{j}$ was presented in Bardet et al. [3] and De Saporta and Yao [14] for a finite state space $S$, in terms of the spectral radius of a certain matrix.

\section{Transient and null-recurrent regimes}

In this section, we study long time behavior of the process $Y=\left(Y_{t}\right)_{t \geq 0}$ and processes defined in terms of certain functionals of $\left(X_{t}\right)_{t \geq 0}$ under conditions different from Assumption 2 and Assumption 3. In particular, it will be assumed that the stability condition $E_{\pi} a(\cdot)>0$ in Assumption 2(a) does not hold and that instead $E_{\pi} a(\cdot) \leq 0$. By choosing a suitable Lyapunov function as done in Shao [37] it follows that $E_{\pi} a(\cdot)<0$ and $E_{\pi} a(\cdot)=0$ correspond to transience and null recurrence, respectively, for the model (1.1).

It can be shown that if Assumption 1 holds and the stability condition $E_{\pi} a(\cdot)>0$ in Assumption 2(a) is replaced by $E_{\pi} a(\cdot)<0$, then the long time behavior of $Y$ will be determined by the first term in the representation

$$
Y_{t}=Y_{0} e^{-\int_{0}^{t} a\left(X_{r}\right) d r}+\int_{0}^{t} b\left(X_{S}\right) e^{-\int_{s}^{t} a\left(X_{r}\right) d r} d W_{s} .
$$

Consequently, the ergodic theorem gives

$$
\frac{\log \left|Y_{t}\right|}{t} \stackrel{\text { a.s. }}{\rightarrow}-E_{\pi} a(\cdot) \quad \text { as } t \rightarrow \infty .
$$


However, it is not well known how scaled fluctuations

$$
\frac{\log \left|Y_{t}\right|}{\sqrt{t}}+\sqrt{t} E_{\pi} a(\cdot)
$$

behave for the model (1.1) as $t \rightarrow \infty$, and how the regime switching dynamics play a role in that limit. This is the motivation behind the results of the present section.

Assumption 4. The $S$-valued process $X$ and the functions $a, b: S \rightarrow \mathbb{R}$ satisfy

(a) $a$ is integrable with respect to $\pi$, and $E_{\pi} a(\cdot) \leq 0$.

(b) For every $j \in S, \sigma_{j}^{2}:=\operatorname{Var}\left(\int_{\tau_{0}^{j}}^{\tau_{1}^{j}}\left(a\left(X_{s}\right)-E_{\pi} a(\cdot)\right) d s\right) \in(0, \infty)$.

(c) Assumption 2(b) holds.

Assumption 4(b) holds trivially for $|S|=2$ or for very simple cyclic Markov chains, but is needed in the general setting for establishing the central-limit-theorem type results in Theorem 2 below.

Theorem 2. Suppose Assumptions 1 and 4 hold. Let $U \sim \pi$ and $N \sim \mathrm{N}(0,1)$ be independent.

(a) (Transient regime) If $E_{\pi} a(\cdot)<0$, then

$$
\frac{\log \left|Y_{t}\right|}{\sqrt{t}}+\sqrt{t} E_{\pi} a(\cdot) \stackrel{d}{\rightarrow} \sum_{j \in S} \delta_{U}(\{j\}) \frac{\sigma_{j}}{\sqrt{E\left|I_{1}^{j}\right|}} N \quad \text { as } t \rightarrow \infty .
$$

(b) (Null-recurrent regime) If $E_{\pi} a(\cdot)=0, b \neq 0$ and

$$
E\left[\left(\log \int_{\tau_{0}^{j}}^{\tau_{1}^{j}} b^{2}\left(X_{S}\right) e^{-2 \int_{s}^{\tau_{1}^{j}} a\left(X_{r}\right) d r} d s\right)^{2}\right]<\infty,
$$

then

$$
\frac{\log \left|Y_{t}\right|}{\sqrt{t}} \stackrel{d}{\rightarrow} \sum_{j \in S} \delta_{U}(\{j\}) \frac{\sigma_{j}}{\sqrt{E\left|I_{1}^{j}\right|}}|N| \quad \text { as } t \rightarrow \infty .
$$

The proof of Theorem 2 is found in Sections 6.5 and 6.6. In Theorem 2(b), $|N|$ appears in the weak limit because the left-hand side in (4.2) asymptotically behaves as a scaled mixture of maxima of partial sums of random walks for which the long time behavior was characterized by Erdös and Kac in [16].

Remark 4.1. The result in Theorem 2(a) does not depend on the diffusion function $b$. In fact, the result holds for any stochastic process $\left(Y_{t}\right)_{t \geq 0}$ such that

$$
Y_{t}=\widetilde{Y}_{t} e^{-\int_{0}^{t} a\left(X_{r}\right) d r} \quad \text { where } \widetilde{Y}_{t}^{1 / \sqrt{t}} \stackrel{d}{\rightarrow} \delta_{1} \text { as } t \rightarrow \infty .
$$


An example is

$$
\widetilde{Y}_{t}=Y_{0}+\int_{0}^{t} b\left(X_{s}\right) e^{\int_{0}^{s} a\left(X_{r}\right) d r} d L_{s}
$$

for a Lévy process $L$ together with an associated integrability condition.

For the exponential integral process $\left(F_{t}\right)_{t \geq 0}$ in (3.7) results similar to Theorem 2 hold. Similar results are found in Theorem 2(a) and Theorem 3(a) in Hitczenko and Wesolowski [26].

Assumption 5. The $S$-valued process $X$ and the functions $c, d: S \rightarrow \mathbb{R}$ satisfy

(a) $c$ is integrable with respect to $\pi$, and $E_{\pi} c(\cdot) \leq 0$.

(b) For every $j \in S, \sigma_{j}^{2}:=\operatorname{Var}\left(\int_{\tau_{0}^{j}}^{\tau_{1}^{j}}\left(c\left(X_{s}\right)-E_{\pi} c(\cdot)\right) d s\right) \in(0, \infty)$.

(c) Assumption 3(b) holds.

Proposition 4.2. Suppose that Assumptions 1 and 5 hold. Let $U \sim \pi$ and $N \sim \mathrm{N}(0,1)$ be independent.

(a) (Transient regime) If $E_{\pi} c(\cdot)<0$, then

$$
\frac{\log \left|F_{t}\right|}{\sqrt{t}}+\sqrt{t} E_{\pi} c(\cdot) \stackrel{d}{\rightarrow} \sum_{j \in S} \delta_{U}(\{j\}) \frac{\sigma_{j}}{\sqrt{E\left|I_{1}^{j}\right|}} N \quad \text { as } t \rightarrow \infty .
$$

(b) (Null-recurrent regime) If $E_{\pi} c(\cdot)=0, d \neq 0$ and

$$
E\left[\left(\log ^{+} \int_{\tau_{0}^{j}}^{\tau_{1}^{j}} d\left(X_{s}\right) e^{-\int_{s}^{\tau_{k}^{j}} c\left(X_{r}\right) d r} d s\right)^{2}\right]<\infty
$$

then

$$
\frac{\log \left|F_{t}\right|}{\sqrt{t}} \stackrel{d}{\rightarrow} \sum_{j \in S} \delta_{U}(\{j\}) \frac{\sigma_{j}}{\sqrt{E\left|I_{1}^{j}\right|}}|N| \quad \text { as } t \rightarrow \infty .
$$

Proposition 4.2 can be proved by a minor modification of the proof of Theorem 2 . The proof of Proposition 4.2 is therefore omitted.

\section{Applications}

The results presented in Sections 3 and 4 have applications in various contexts involving stochastic processes under hidden Markovian environments. We consider two specific applications. 


\subsection{The Cox-Ingersoll-Ross process}

The Cox-Ingersoll-Ross (CIR) model is a stochastic differential equation introduced in Cox, Ingersoll and Ross [13] as a model for interest rates (short rates) that has been extensively applied since then. In Hou and Shao [27] criteria for existence of a stationary distribution of a CIR model with regime-switching coefficients were determined and tail behavior was analyzed in terms of existence of moments. In this section, we consider a natural parametrization of the CIR model with parameters fluctuating according to a hidden Markov chain and derive explicit expressions for its exact long time behavior. From these explicit expressions the tail behavior of the regimeswitching CIR model can be determined.

Let $a, b: S \rightarrow \mathbb{R}$, with $a(x) \neq 0$ for all $x \in S$, be arbitrary functions, let $n \in \mathbb{N}$ such that $n \geq 2$, and define

$$
\kappa, \theta, \xi: S \rightarrow \mathbb{R}, \quad \kappa:=2 a, \theta:=\frac{n b^{2}}{2 a}, \xi:=2 b .
$$

Consider the CIR process $\left(R_{t}\right)_{t \geq 0}$ defined as the solution to the stochastic differential equation

$$
d R_{t}=\kappa\left(X_{t}\right)\left(\theta\left(X_{t}\right)-R_{t}\right) d t+\xi\left(X_{t}\right) \sqrt{R_{t}} d W_{t}, \quad R_{0}=r_{0}>0 .
$$

The condition $2 \kappa \theta \geq \xi^{2}$, which holds automatically for the above parametrization since $n \geq 2$ regardless of the value of $X_{t}=x \in S$, ensures that the CIR model with probability 1 takes only strictly positive values.

Proposition 5.1. Consider the CIR model (5.1) with the above parametrization and $X$ as defined in (1.2). Let $U \sim \pi$ and $N_{1}, \ldots, N_{n} \sim \mathrm{N}(0,1)$ be independent.

(a) Suppose Assumptions 1 and 2 hold $\left(E_{\pi} \kappa(\cdot)>0\right.$ and integrability of $\left.\xi\right)$. Then

$$
R_{t} \stackrel{d}{\rightarrow} \sum_{j \in S} \delta_{U}(\{j\}) V_{j} \sum_{i=1}^{n} N_{i}^{2} \quad \text { as } t \rightarrow \infty
$$

where $V_{j}$ is independent of $U, N_{1}, \ldots, N_{n}$ and $\mathcal{L}\left(V_{j}\right)$ is given in Theorem 1.

(b) Suppose Assumptions 1 and 4 hold. If $E_{\pi} \kappa(\cdot)<0$, then

$$
\frac{\log R_{t}}{\sqrt{t}}+\sqrt{t} E_{\pi} \kappa(\cdot) \stackrel{d}{\rightarrow} \sum_{j \in S} \delta_{U}(\{j\}) \frac{2 \sigma_{j}}{\sqrt{E\left|I_{1}^{j}\right|}} N \quad \text { as } t \rightarrow \infty .
$$

(c) Suppose Assumptions 1 and 4 hold. If $E_{\pi} \kappa(\cdot)=0, \xi \neq 0$ and (4.1) holds, then

$$
\frac{\log R_{t}}{\sqrt{t}} \stackrel{d}{\rightarrow} \sum_{j \in S} \delta_{U}(\{j\}) \frac{2 \sigma_{j}}{\sqrt{E\left|I_{1}^{j}\right|}}|N| \quad \text { as } t \rightarrow \infty .
$$

The proof of Proposition 5.1 is found in the supplementary material [30].

Notice that the limit laws in Proposition 5.1(b) and (c) do not depend on $n$. We conjecture that part (b) and (c) hold also for noninteger $n$. For noninteger $n$, the solution to the CIR process can 
be written as a sum of a squared Ornstein-Uhlenbeck processes and a squared Bessel process (Chapter 6 in Jeanblanc et al. [28]). Therefore, one needs to investigate long time behavior of Bessel process under Markovian regime switching in order to generalize Proposition 5.1 further.

\subsection{The SIS model in epidemiology}

We consider deterministic SIS epidemic models under Markov modulated environments similar to the one considered in Gray et al. [22], but with $|S| \geq 2$ number of regimes instead of just two. Let $\alpha, \beta: S \rightarrow(0, \infty)$ be functions denoting the rate of infection and recovery, respectively. Consider a fixed population of size $n$ and subpopulation sizes $I_{t}$ and $S_{t}$ at times $t \geq 0$, satisfying $I_{t}+S_{t}=n$, of infectious and susceptible individuals, respectively. The model is determined by the system of equations

$$
\frac{d S_{t}}{d t}=-\beta\left(X_{t}\right) S_{t} I_{t}+\alpha\left(X_{t}\right) I_{t}, \quad \frac{d I_{t}}{d t}=\beta\left(X_{t}\right) S_{t} I_{t}-\alpha\left(X_{t}\right) I_{t}, \quad I_{t}+S_{t}=n, I_{0}>0,
$$

where $I_{t}, S_{t}$ may take arbitrary real values in $[0, n]$. If $\alpha, \beta>0$ were constants instead of functions, then one would have, with $\gamma:=\beta n-\alpha$,

$$
I_{t}=\left[e^{-\gamma t}\left(\frac{1}{I_{0}}-\frac{\beta}{\gamma}\right)+\frac{\beta}{\gamma}\right]^{-1} 1_{\{\gamma \neq 0\}}+\left[\frac{1}{I_{0}}+\beta t\right]^{-1} 1_{\{\gamma=0\}}
$$

which would lead to

$$
\lim _{t \rightarrow \infty} I_{t}=\frac{\gamma}{\beta} \text { if } \gamma>0, \quad \lim _{t \rightarrow \infty} I_{t}=0 \quad \text { if } \gamma \leq 0
$$

Notice that $\gamma \leq 0$ is equivalent to $R_{0} \leq 1$, where $R_{0}$ is the reproduction number. For $|S|=2$, results from Gray et al. [22] suggest the following:

(a) If $E_{\pi} \gamma(\cdot)>0$, then persistence holds for the infected population size:

$$
\liminf _{t \rightarrow \infty} I_{t} \leq \frac{E_{\pi} \gamma(\cdot)}{E_{\pi} \beta(\cdot)} \quad \text { a.s., } \quad \limsup _{t \rightarrow \infty} I_{t} \geq \frac{E_{\pi} \gamma(\cdot)}{E_{\pi} \beta(\cdot)} \quad \text { a.s. }
$$

(b) For $E_{\pi} \gamma(\cdot)=0$ it is conjectured that $I_{t} \rightarrow 0$ in probability (Example 6.3.1 in [22] supported by a simulation study).

(c) If $E_{\pi} \gamma(\cdot)<0$, then $I_{t} \rightarrow 0$ exponentially fast: $\limsup _{t \rightarrow \infty}\left(\log I_{t}\right) / t \leq E_{\pi} \gamma(\cdot)$ a.s.

The following proposition provides sharper asymptotic distributional results.

Proposition 5.2. Suppose that Assumption 3(b) holds with $(c, d)=(\gamma, \beta)$. For the model described above the following statements hold:

(a) If $E_{\pi} \gamma(\cdot)>0$, then for $a, b \in \mathbb{R}$ with $a<b$,

$$
\lim _{t \rightarrow \infty} P\left[\frac{1}{I_{t}} \in(a, b)\right]=\sum_{j \in S} \pi_{j} P\left[V_{j} \in(a, b)\right],
$$

where $V_{j}$ is given in Proposition 3.7 when $(c, d)=(\gamma, \beta)$. 
(b) If $E_{\pi} \gamma(\cdot)=0, \beta \neq 0$, (4.3) holds with $(c, d)=(\gamma, \beta)$, and $\sigma_{j}^{2}:=\operatorname{Var}\left(\int_{\tau_{0}^{j}}^{\tau_{1}^{j}} \gamma\left(X_{s}\right) d s\right) \in$ $(0, \infty)$, then, for $a, b \in \mathbb{R}$ with $0 \leq a<b$ and $N \sim \mathrm{N}(0,1)$,

$$
\lim _{t \rightarrow \infty} P\left[I_{t} \in\left(e^{-b \sqrt{t}}, e^{-a \sqrt{t}}\right)\right]=\sum_{j \in S} \pi_{j} P\left[|N| \in \frac{\sqrt{E\left|I_{1}^{j}\right|}}{\sigma_{j}}(a, b)\right] .
$$

(c) If $E_{\pi} \gamma(\cdot)<0$ and $\sigma_{j}^{2}:=\operatorname{Var}\left(\int_{\tau_{0}^{j}}^{\tau_{1}^{j}}\left(\gamma\left(X_{s}\right)-E_{\pi} \gamma(\cdot)\right) d s\right) \in(0, \infty)$, then, for $a, b \in \mathbb{R}$ with $a<b$ and $N \sim \mathrm{N}(0,1)$,

$$
\lim _{t \rightarrow \infty} P\left[I_{t} \in\left(e^{t E_{\pi} \gamma(\cdot)-b \sqrt{t}}, e^{t E_{\pi} \gamma(\cdot)-a \sqrt{t}}\right)\right]=\sum_{j \in S} \pi_{j} P\left[N \in \frac{\sqrt{E\left|I_{1}^{j}\right|}}{\sigma_{j}}(a, b)\right] .
$$

The proof of Proposition 5.2 is found in the supplementary material [30].

Similar results can be obtained for Markov modulated deterministic SIR models as integrals of type $\int_{0}^{t} e^{-\int_{s}^{t} \gamma\left(X_{r}\right) d r} \beta\left(X_{s}\right) d s$ show up as a consequence of the Markovian environment in the transition rates.

\section{Proofs}

We use the convention $\sum_{i=j}^{k} a_{i}=0$ and $\prod_{i=j}^{k} a_{i}=1$ if $j>k$ for any $a_{i}$. For functions $c, d$ : $S \rightarrow \mathbb{R}$ and $j \in S$, define

$$
G_{j}^{c, d}(x):=\int_{0}^{x} d(j) e^{-c(j)(x-s)} d s=x d(j) 1_{\{c(j)=0\}}+\frac{d(j)}{c(j)}\left(1-e^{-x c(j)}\right) 1_{\{c(j) \neq 0\}} .
$$

\subsection{Proof of Theorem 1}

Proof. We prove the statement in a number of steps. The marginal distribution of the regime process $X$ in $S$ with initial distribution $\delta_{i}, i \in S$, is denoted

$$
P_{i j}(0, t):=P\left[X_{t}=j \mid X_{0}=i\right]=P_{t}(i,\{j\}) .
$$

Let the transition kernels of the time-homogeneous Markov process $\left(X_{t}, Y_{t}\right)_{t \geq 0}$ be the maps $\mathbf{P}^{t}:(S \times \mathbb{R}) \times \mathcal{B}(S \times \mathbb{R}) \rightarrow[0,1]$. Then, for any $\left(i, y_{0}\right) \in S \times \mathbb{R}$ and $I \times A \in \mathcal{B}(S \times \mathbb{R})$,

$$
\begin{aligned}
\mathbf{P}^{t}\left(\left(i, y_{0}\right), I \times A\right) & =\sum_{j \in I} P\left[\left(X_{t}, Y_{t}\right) \in(\{j\}, A) \mid\left(X_{0}, Y_{0}\right)=\left(i, y_{0}\right)\right] \\
& =\sum_{j \in I} P_{i j}(0, t) P\left[Y_{t} \in A \mid Y_{0}=y_{0}, X_{0}=i, X_{t}=j\right],
\end{aligned}
$$


since, by (1.3),

$$
P\left[X_{t}=j \mid\left(X_{0}, Y_{0}\right)=\left(i, y_{0}\right)\right]=P\left[X_{t}=j \mid X_{0}=i\right]=P_{i j}(0, t) .
$$

If we can show that there exists a $\mu_{\infty} \in \mathcal{P}(S \times R)$ such that

$$
\delta_{\left(i, y_{0}\right)} \mathbf{P}^{t} \stackrel{w}{\rightarrow} \mu_{\infty} \quad \text { as } t \rightarrow \infty \text { for all }\left(i, y_{0}\right) \in(S \times \mathbb{R}),
$$

then as a consequence of the strong Feller property, satisfied trivially by $(X, Y)$, one can deduce that $\mu_{\infty}$ is the unique invariant distribution. The strong Feller property ensures that one may interchange the order of limit and expectation in

$$
\mu_{\infty} \mathbf{P}^{s} f=\lim _{t \rightarrow \infty} \delta_{\left(i, y_{0}\right)} \mathbf{P}^{t} \mathbf{P}^{s} f=\lim _{t \rightarrow \infty} \delta_{\left(i, y_{0}\right)} \mathbf{P}^{t+s} f=\mu_{\infty} f \quad \text { for all } s>0 .
$$

We find $\mu_{\infty}$ using (6.3).

Lemma 6.1 below provides a representation of the second factor in the product in (6.2). The representation is expressed in terms of an $\left(\mathcal{F}_{t}^{X}\right)_{t \geq 0}$-adapted stochastic process $\left(Q_{t}^{(1)}, Q_{t}^{(2)}\right)_{t \geq 0}$. Fix two arbitrary states $i, j \in S$ and suppose that $X_{0}=i$. Recall that $\tau_{0}^{j}$ is the first time $X$ visits state $j$ and define $\tau_{k}^{j}$ and $T_{k}^{j}$ for $k \geq 0$ recursively as in (2.3). For $j \in S$, define

$$
\begin{aligned}
& \left(J_{k}^{j}, K_{k}^{j}\right):=\left(e^{-\int_{\tau_{k-1}^{j}}^{\tau_{k}^{j}} a\left(X_{s}\right) d s}, \int_{\tau_{k-1}^{j}}^{\tau_{k}^{j}} b^{2}\left(X_{s}\right) e^{-2 \int_{s}^{\tau_{k}^{j}} a\left(X_{r}\right) d r} d s\right), \quad k \geq 1, \\
& \left(J_{0}^{j}, K_{0}^{j}\right):=\left(e^{-\int_{0}^{\tau_{0}^{j}} a\left(X_{s}\right) d s}, \int_{0}^{\tau_{0}^{j}} b^{2}\left(X_{s}\right) e^{-2 \int_{s}^{\tau_{0}^{j}} a\left(X_{r}\right) d r} d s\right) .
\end{aligned}
$$

Notice that if $X_{0}=j$, then $\tau_{0}^{j}=0$ and $\left(J_{0}^{j}, K_{0}^{j}\right)=(1,0)$. The sequence $\left(I_{k}^{j}\right)_{k \geq 1}$ of renewal cycles is an i.i.d. sequence and therefore also $\left(J_{k}^{j}, K_{k}^{j}\right)_{k=1}^{\infty}$ is an i.i.d. sequence. However, for a fixed $t>0,\left(J_{k}^{j}, K_{k}^{j}\right)_{k=1}^{g_{t}^{j}}$ is not an i.i.d. sequence since $g_{t}^{j}$, defined in (2.4), is a renewal time which depends on the sum of all renewal cycle lengths before time $t$.

Lemma 6.1. Suppose Assumptions 1 and 2 hold. Then, for any $t>0$ and $A \in \mathcal{B}(\mathbb{R})$,

$$
P\left[Y_{t} \in A \mid Y_{0}=y_{0}, X_{0}=i, X_{t}=j\right]=P\left[y_{0} Q_{t}^{(1)}+\sqrt{Q_{t}^{(2)}} N \in A \mid X_{0}=i, X_{t}=j\right],
$$

where $N \sim \mathrm{N}(0,1), N \Perp\left(\mathcal{F}_{t}^{X}\right)_{t \geq 0}$ and $\left(Q_{t}^{(1)}, Q_{t}^{(2)}\right)_{t \geq 0}$ is an $\left(\mathcal{F}_{t}^{X}\right)_{t \geq 0}$-adapted process given by

$$
\begin{aligned}
& Q_{t}^{(1)}=e^{-a(j)\left(t-\tau^{j}{ }^{j}\right)}\left(\prod_{k=1}^{g_{t}^{j}} J_{k}^{j}\right) J_{0}^{j}, \\
& Q_{t}^{(2)}=G_{j}^{2 a, b^{2}}\left(t-\tau_{g_{t}^{j}}^{j}\right)+e^{-2 a(j)\left(t-\tau_{j}^{j}{ }_{j}{ }^{j}\right)}\left[\left(\prod_{k=1}^{g_{t}^{j}} J_{k}^{j}\right)^{2} K_{0}^{j}+\sum_{k=1}^{g_{t}^{j}}\left(\prod_{l=k+1}^{g_{t}^{j}} J_{l}^{j}\right)^{2} K_{k}^{j}\right] .
\end{aligned}
$$


The proof of Lemma 6.1 is found in Section 6.2.

Now, using Lemma 6.1 , we prove the main result by finding $\mu_{\infty}$ by computing the limit as $t \rightarrow \infty$ for a term in the sum (6.2). Note that $P_{i j}(0, t) \rightarrow \pi_{j}$ as $t \rightarrow \infty$ by the ergodicity property in Assumption 1. Using Lemma 6.1 it remains to show that

$$
P\left[y_{0} Q_{t}^{(1)}+\sqrt{Q_{t}^{(2)}} N \in A \mid X_{0}=i, X_{t}=j\right] \rightarrow P\left[\sqrt{V_{j}} N \in A\right], \quad(A, j) \in \mathcal{B}(\mathbb{R}) \times S .
$$

Let us summarize the steps. (6.5) follows if we show

$$
\mathcal{L}\left(\left(Q_{t}^{(1)}, Q_{t}^{(2)}\right) \mid X_{0}=i, X_{t}=j\right) \stackrel{w}{\rightarrow} \delta_{0} \otimes \mathcal{L}\left(V_{j}\right) \quad \text { as } t \rightarrow \infty .
$$

The result above is observed by taking the limit as $t \rightarrow \infty$ of the expression on the right-hand side of (6.4). Set

$$
R_{t}:=\left(R_{t}^{(1)}, R_{t}^{(2)}\right):=\left(\left(\prod_{k=1}^{g_{t}^{j}} J_{k}^{j}\right) J_{0}^{j},\left(\prod_{k=1}^{g_{t}^{j}} J_{k}^{j}\right)^{2} K_{0}^{j}+\sum_{k=1}^{g_{t}^{j}}\left(\prod_{l=k+1}^{g_{t}^{j}} J_{l}^{j}\right)^{2} K_{k}^{j}\right)
$$

and notice that, on $\left\{X_{0}=i, X_{t}=j\right\}$,

$$
\left(Q_{t}^{(1)}, Q_{t}^{(2)}\right)=\left(e^{-a(j)\left(t-\tau^{j}{ }^{j}\right)} R_{t}^{(1)}, G_{j}^{2 a, b^{2}}\left(t-\tau_{g_{t}^{j}}^{j}\right)+e^{-2 a(j)\left(t-\tau^{j}{ }^{j}\right)} R_{t}^{(2)}\right) .
$$

Next we determine the weak limit, as $t \rightarrow \infty$, of $\mathcal{L}\left(\left(\left(t-\tau_{g_{t}^{j}}^{j}\right), R_{t}\right) \mid X_{0}=i, X_{t}=j\right)$ and in particular show that $\left(t-\tau_{g_{t}^{j}}^{j}\right.$ ) and $R_{t}$ are asymptotically independent, given $\left\{X_{0}=i, X_{t}=j\right\}$. Take $x \in(0, t)$ and set $B_{t, x}:=\{X$ makes no jump in $(t-x, t]\}$. Then,

$$
\left\{X_{t}=j, t-\tau_{g_{t}^{j}}^{j}>x, R_{t} \in A\right\}=\left\{B_{t, x}, X_{t-x}=j, R_{t-x} \in A\right\}, \quad A:=A_{1} \times A_{2} \in \mathcal{B}\left(\mathbb{R}^{2}\right),
$$

since $\tau_{g_{t}^{j}}^{j}<t-x$ implies $\tau_{g_{t-x}^{j}}^{j}=\tau_{g_{t}{ }^{j}}^{j}$ which further implies $R_{t}=R_{t-x}$. Using the above equality,

$$
\begin{aligned}
P[t & \left.-\tau_{g_{t}^{j}}^{j}>x, R_{t} \in A \mid X_{0}=i, X_{t}=j\right] \\
= & P\left[B_{t, x}, X_{t-x}=j, R_{t-x} \in A \mid X_{0}=i, X_{t}=j\right] \\
= & P\left[B_{t, x} \mid X_{t-x}=j, R_{t-x} \in A, X_{0}=i\right] P\left[R_{t-x} \in A \mid X_{t-x}=j, X_{0}=i\right] \\
& \quad \times \frac{P\left[X_{t-x}=j, X_{0}=i\right]}{P\left[X_{t}=j, X_{0}=i\right]} .
\end{aligned}
$$

Since $R_{t-x}$ is $\mathcal{F}_{t-x}^{X}$-measurable, the Markov property of $X$ implies

$$
P\left[B_{t, x} \mid X_{t-x}=j, R_{t-x} \in A, X_{0}=i\right]=P\left[B_{t, x} \mid X_{t-x}=j\right]=e^{\lambda_{j j} x} .
$$


Moreover,

$$
\lim _{t \rightarrow \infty} \frac{P\left[X_{t-x}=j, X_{0}=i\right]}{P\left[X_{t}=j, X_{0}=i\right]}=1 .
$$

It remains to show the existence of

$$
\lim _{t \rightarrow \infty} P\left[R_{t-x} \in A \mid X_{t-x}=j, X_{0}=i\right]=\lim _{t \rightarrow \infty} P\left[R_{t-x} \in A \mid X_{t-x}=j\right]
$$

and determine the limit explicitly. Clearly, if the limit exists, then it coincides with $\lim _{t \rightarrow \infty} P\left[R_{t} \in A \mid X_{t}=j\right]$. We will show the convergence by first proving (in Lemma 6.2 below) that $\mathcal{L}\left(R_{t}\right) \stackrel{w}{\rightarrow} \mathcal{L}\left(R_{\infty}\right)$ as $t \rightarrow \infty$ and determine $\mathcal{L}\left(R_{\infty}\right)$, and then (by combining Lemmas 6.3 and 6.4 below) prove that

$$
\mathcal{L}\left(R_{t}\right) \stackrel{w}{\rightarrow} \mathcal{L}\left(R_{\infty}\right) \quad \text { as } t \rightarrow \infty \quad \text { implies } \quad \mathcal{L}\left(R_{t} \mid X_{t}=j\right) \stackrel{w}{\rightarrow} \mathcal{L}\left(R_{\infty}\right) \quad \text { as } t \rightarrow \infty
$$

where $\mathcal{L}\left(R_{\infty}\right)$ is described as $\mathcal{L}\left(0, V_{j}^{*}\right)$ in Lemma 6.2. Notice that once the latter convergence is shown, the asymptotic independence of $\left(t-\tau_{g_{t}^{j}}^{j}\right)$ and $R_{t}$ given $X_{t}=j$ is established from (6.9) and that $\mathcal{L}\left(t-\tau_{g_{t}^{j}}^{j} \mid X_{t}=j\right) \stackrel{w}{\rightarrow} \operatorname{Exp}\left(-\lambda_{j j}\right)$ as ${ }_{t} \rightarrow \infty$.

Recall that $R_{t}$ in (6.7) is defined for an arbitrary but fixed $j \in S$.

Lemma 6.2. Under Assumptions 1 and $2, R_{t}$ in (6.7) satisfies $R_{t} \stackrel{d}{\rightarrow}\left(0, V_{j}^{*}\right)$ as $t \rightarrow \infty$, where $V_{j}^{*}$ satisfies

$$
\begin{aligned}
& \sum_{k=1}^{g_{t}^{j}}\left(\prod_{l=k+1}^{g_{t}^{j}} J_{l}^{j}\right)^{2} K_{k}^{j} \stackrel{d}{\rightarrow} V_{j}^{*} \quad \text { as } t \rightarrow \infty, \\
& V_{j}^{*} \stackrel{d}{=}\left(J_{1}^{j}\right)^{2} V_{j}^{*}+K_{1}^{j}, \quad V_{j}^{*} \Perp\left(J_{1}^{j}, K_{1}^{j}\right) .
\end{aligned}
$$

The proof of Lemma 6.2 is found in Section 6.3.

Lemma 6.3. Consider an $\left(\mathcal{F}_{t}^{X}\right)_{t \geq 0}$-adapted process $\left(H_{t}\right)_{t \geq 0}$, and suppose there exist a random variable $H_{\infty}$ and an increasing function $t \mapsto \varepsilon(t)$ such that

$$
H_{t} \stackrel{d}{\rightarrow} H_{\infty}, \quad \varepsilon(t) \rightarrow \infty, \quad \frac{\varepsilon(t)}{t} \rightarrow 0 \quad \text { and } \quad H_{t}-H_{t-\varepsilon(t)} \stackrel{P}{\rightarrow} 0 \quad \text { as } t \rightarrow \infty
$$

Suppose further that $\left(L_{t}^{(1)}, L_{t}^{(2)}\right)_{t \geq 0}$ satisfies $\left(L_{t}^{(1)}, L_{t}^{(2)}\right) \stackrel{P}{\rightarrow}(0,1)$ as $t \rightarrow \infty$. Then, for any $A$ such that $P\left[H_{\infty} \in \partial A\right]=0$,

$$
\lim _{t \rightarrow \infty} P\left[L_{t}^{(1)}+L_{t}^{(2)} H_{t} \in A \mid X_{t}=j\right]=P\left[H_{\infty} \in A\right] .
$$

The proof of Lemma 6.3 is found in Section 6.4. 
Lemma 6.4. There exists an $\left(\mathcal{F}_{t}^{X}\right)_{t \geq 0}$-adapted process $\left(R_{t}^{*}\right)_{t \geq 0}$ such that $\left(R_{t}\right)_{t \geq 0}$ in (6.7) satisfies $\mathcal{L}\left(R_{t}^{*}\right)=\mathcal{L}\left(R_{t}\right)$ and $\mathcal{L}\left(R_{t}^{*} \mid X_{t}=j\right)=\mathcal{L}\left(R_{t} \mid X_{t}=j\right)$, and $\left(R_{t}^{*}\right)_{t \geq 0}$ satisfies the conditions for $\left(H_{t}\right)_{t \geq 0}$ in (6.11) in Lemma 6.3 with $\left(0, V_{j}^{*}\right)$ as $H_{\infty}$.

The proof of Lemma 6.4 is found in the supplementary material [30].

Notice that for establishing weak convergence it is sufficient to restrict attention to continuity sets, that is, here sets satisfying $P\left[H_{\infty} \in \partial A\right]=0$. The part of (6.11) involving $\varepsilon(t)$ resembles Anscombe's condition (Gut [23], page 16).

Using Lemmas 6.2, 6.3 and 6.4 together with (6.8) prove that

$$
\mathcal{L}\left(\left(Q_{t}^{(1)}, Q_{t}^{(2)}\right) \mid X_{0}=i, X_{t}=j\right) \stackrel{w}{\rightarrow} \delta_{0} \otimes \mathcal{L}\left(G_{j}^{2 a, b^{2}}\left(T^{j}\right)+e^{-2 a(j) T^{j}} V_{j}^{*}\right) \quad \text { as } t \rightarrow \infty
$$

where $T^{j} \sim \operatorname{Exp}\left(-\lambda_{j j}\right)$ is independent of $V_{j}^{*}$. This proves (6.6) and concludes the proof of Theorem 1.

\subsection{Proof of Lemma 6.1}

Proof. The process $\left(Y_{t}\right)_{t \geq 0}$ in (1.1) has the representation

$$
Y_{t}=\boldsymbol{\Phi}(0, t) Y_{0}+\int_{0}^{t} b\left(X_{s}\right) \boldsymbol{\Phi}(s, t) d W_{s}, \quad \boldsymbol{\Phi}(s, t):=e^{-\int_{s}^{t} a\left(X_{r}\right) d r} .
$$

Define

$$
\left(Q_{t}^{(1)}, Q_{t}^{(2)}\right):=\left(\boldsymbol{\Phi}(0, t), \int_{0}^{t} b^{2}\left(X_{s}\right) \boldsymbol{\Phi}^{2}(s, t) d s\right) .
$$

Note that on $\left\{X_{0}=i, X_{t}=j\right\}:=\left\{\omega \in \Omega: X_{0}(\omega)=i, X_{t}(\omega)=j\right\}$,

$$
\begin{aligned}
Q_{t}^{(1)} & =\boldsymbol{\Phi}(0, t)=\boldsymbol{\Phi}\left(\tau_{g_{t}^{j}}^{j}, t\right)\left(\prod_{l=1}^{g_{t}^{j}} \boldsymbol{\Phi}\left(\tau_{l-1}^{j}, \tau_{l}^{j}\right)\right) \boldsymbol{\Phi}\left(0, \tau_{0}^{j}\right) \\
& =e^{-a(j)\left(t-\tau_{g_{t}^{j}}^{j}\right)}\left(\prod_{l=1}^{g_{t}^{j}} J_{l}^{j}\right) J_{0}^{j},
\end{aligned}
$$

where the identity $\boldsymbol{\Phi}\left(\tau_{g_{t}^{j}}^{j}, t\right)=e^{-a(j)\left(t-\tau^{j}{ }^{j}\right)}$ on $\left\{X_{0}=i, X_{t}=j\right\}$ follows from the fact that if $s \in\left[\tau_{g_{t}^{j}}^{j}, t\right)$, then $X_{s}=j$.

We now consider the second term in (6.12). Notice that by Itô isometry

$$
\mathcal{L}\left(\int_{0}^{t} b\left(X_{s}\right) \boldsymbol{\Phi}(s, t) d W_{s} \mid X_{0}=i, X_{t}=j\right)
$$




$$
=\mathcal{L}\left(\left[\int_{0}^{t} b^{2}\left(X_{S}\right) \boldsymbol{\Phi}^{2}(s, t) d s\right]^{1 / 2} N \mid X_{0}=i, X_{t}=j\right) .
$$

Partitioning $[0, t]$ into $[0, t]=\left[0, \tau_{0}^{j}\right) \cup \bigcup_{k=1}^{g_{t}^{j}}\left[\tau_{k-1}^{j}, \tau_{k}^{j}\right) \cup\left[\tau_{g_{t}^{j}}^{j}, t\right]$, we may write $Q_{t}^{(2)}$ as

$$
\begin{aligned}
\int_{0}^{t} b^{2}\left(X_{S}\right) \boldsymbol{\Phi}^{2}(s, t) d s= & \int_{0}^{\tau_{0}^{j}} b^{2}\left(X_{S}\right) \boldsymbol{\Phi}^{2}(s, t) d s+\sum_{k=1}^{g_{t}^{j}} \int_{\tau_{k-1}^{j}}^{\tau_{k}^{j}} b^{2}\left(X_{S}\right) \boldsymbol{\Phi}^{2}(s, t) d s \\
& +\int_{\substack{\tau_{j}^{j} j \\
g_{t}}}^{t} b^{2}\left(X_{S}\right) \boldsymbol{\Phi}^{2}(s, t) d s .
\end{aligned}
$$

Expanding one term in the middle sum

$$
\begin{aligned}
\int_{\tau_{k-1}^{j}}^{\tau_{k}^{j}} b^{2}\left(X_{s}\right) \boldsymbol{\Phi}^{2}(s, t) d s & =\int_{\tau_{k-1}^{j}}^{\tau_{k}^{j}} \boldsymbol{\Phi}^{2}\left(\tau_{k}^{j}, t\right) b^{2}\left(X_{s}\right) \boldsymbol{\Phi}^{2}\left(s, \tau_{k}^{j}\right) d s \\
& =\boldsymbol{\Phi}^{2}\left(\tau_{g_{t}^{j}}^{j}, t\right)\left(\prod_{i=k}^{g_{t}^{j}-1} \boldsymbol{\Phi}^{2}\left(\tau_{i}^{j}, \tau_{i+1}^{j}\right)\right) \int_{\tau_{k-1}^{j}}^{\tau_{k}^{j}} b^{2}\left(X_{s}\right) \boldsymbol{\Phi}^{2}\left(s, \tau_{k}^{j}\right) d s
\end{aligned}
$$

for some $k \leq g_{t}^{j}$. Putting these expressions in (6.15) gives

$$
\begin{aligned}
\int_{0}^{t} b^{2}\left(X_{s}\right) \boldsymbol{\Phi}^{2}(s, t) d s= & \boldsymbol{\Phi}^{2}\left(\tau_{g_{t}^{j}}^{j}, t\right)\left[\left(\prod_{l=0}^{g_{t}^{j}-1} \boldsymbol{\Phi}^{2}\left(\tau_{l}^{j}, \tau_{l+1}^{j}\right)\right) \int_{0}^{\tau_{0}^{j}} b^{2}\left(X_{s}\right) \boldsymbol{\Phi}^{2}\left(s, \tau_{0}^{j}\right) d s\right. \\
& \left.+\sum_{k=1}^{g_{t}^{j}}\left(\prod_{l=k}^{g_{t}^{j}-1} \boldsymbol{\Phi}^{2}\left(\tau_{l}^{j}, \tau_{l+1}^{j}\right)\right) \int_{\tau_{k}^{j}-1}^{\tau_{k}^{j}} b^{2}\left(X_{s}\right) \boldsymbol{\Phi}^{2}\left(s, \tau_{k}^{j}\right) d s\right] \\
& +\int_{\tau_{g_{t}^{j}}^{j}}^{t} b^{2}\left(X_{s}\right) \boldsymbol{\Phi}^{2}(s, t) d s .
\end{aligned}
$$

On $\left\{X_{0}=i, X_{t}=j\right\}$,

$$
\int_{\substack{\tau^{j} \\ g_{t}^{j}}}^{t} b^{2}\left(X_{s}\right) \boldsymbol{\Phi}^{2}(s, t) d s=b^{2}(j) \int_{\tau^{j}{ }_{t}^{j}}^{t} e^{-2(t-s) a(j)} d s=G_{j}^{2 a, b^{2}}\left(t-\tau_{g_{t}^{j}}^{j}\right) .
$$

Moreover, on $\left\{X_{0}=i, X_{t}=j\right\}$,

$$
\int_{0}^{\tau^{j} g_{t}^{j}} b^{2}\left(X_{S}\right) \boldsymbol{\Phi}^{2}(s, t) d s=e^{-2 a(j)\left(t-\tau^{j}{ }^{j}\right)}\left[\left(\prod_{k=1}^{g_{t}^{j}} J_{k}^{j}\right)^{2} K_{0}^{j}+\sum_{k=1}^{g_{t}^{j}}\left(\prod_{l=k+1}^{g_{t}^{j}} J_{l}^{j}\right)^{2} K_{k}^{j}\right] .
$$


Combining the above gives, on $\left\{X_{0}=i, X_{t}=j\right\}$,

$$
Q_{t}^{(2)}=G_{j}^{2 a, b^{2}}\left(t-\tau_{g_{t}^{j}}^{j}\right)+e^{-2 a(j)\left(t-\tau^{j}{ }^{j}\right)}\left[\left(\prod_{k=1}^{g_{t}^{j}} J_{k}^{j}\right)^{2} K_{0}^{j}+\sum_{k=1}^{g_{t}^{j}}\left(\prod_{l=k+1}^{g_{t}^{j}} J_{l}^{j}\right)^{2} K_{k}^{j}\right]
$$

The assertion of Lemma 6.1 follows by combining (6.14) and (6.16).

\subsection{Proof of Lemma 6.2}

In this section, we prove Lemma 6.2 by first proving (6.10) and then

$$
\left(\prod_{k=1}^{g_{t}^{j}} J_{k}^{j}\right) J_{0}^{j} \stackrel{d}{\rightarrow} 0, \quad\left(\prod_{k=1}^{g_{t}^{j}} J_{k}^{j}\right)^{2} K_{0}^{j} \stackrel{d}{\rightarrow} 0 \quad \text { as } t \rightarrow \infty
$$

Proof of (6.10). To simplify the notation we omit the superscript $j$ in this subsection. Main technical difficulty here is to obtain a similar version of Vervaat's Theorem 1.5 in [40] but in a continuous time context driven by the renewal time $g_{t}$. For $n \geq 1$, define

$$
\widetilde{S}_{n}:=\sum_{k=1}^{n}\left(\prod_{i=k+1}^{n} J_{i}\right)^{2} K_{k}=K_{n}+J_{n}^{2} K_{n-1}+\cdots+\left(J_{2} \cdots J_{n}\right)^{2} K_{1} .
$$

Note that $\widetilde{S}_{n+1}=\widetilde{S}_{n} J_{n+1}^{2}+K_{n+1}$ and therefore $\left(\widetilde{S}_{n}\right)_{n \geq 1}$ is Markovian. For $n \geq 1$, define

$$
S_{n}:=\sum_{k=1}^{n}\left(\prod_{i=1}^{k-1} J_{i}\right)^{2} K_{k}=K_{1}+J_{1}^{2} K_{2}+\cdots+\left(J_{1} \cdots J_{n-1}\right)^{2} K_{n} .
$$

Note that $S_{n+1}=S_{n}+\left(J_{1}^{2} \ldots J_{n}^{2}\right) K_{n+1}$ and therefore $\left(S_{n}\right)_{n \geq 1}$ is not Markovian but $S_{n}$ is a partial sum of the infinite sum

$$
S_{\infty}:=\sum_{k=1}^{\infty}\left(\prod_{i=1}^{k-1} J_{i}\right)^{2} K_{k}
$$

We will show that $\widetilde{S}_{g_{t}} \stackrel{d}{\rightarrow} S_{\infty}$ as $t \rightarrow \infty$ where $S_{\infty}$ satisfies $S_{\infty} \stackrel{d}{=}\left(J_{1}\right)^{2} S_{\infty}+K_{1}, S_{\infty} \Perp\left(J_{1}, K_{1}\right)$. From uniqueness of the solution we will conclude that $\mathcal{L}\left(S_{\infty}\right)=\mathcal{L}\left(V_{j}^{*}\right)$. We will prove (6.10) in the following steps.

- Step 1: $\widetilde{S}_{g_{t}} \stackrel{d}{=} S_{g_{t}}$ for any $t>0$.

- Step 2: $\frac{\log K_{g_{t+1}}}{g_{t}} \stackrel{\text { a.s. }}{\rightarrow} 0$ as $t \rightarrow \infty$.

- Step 3: $S_{\infty} \stackrel{d}{=} \sum_{k=1}^{\infty}\left(\prod_{i=1}^{k-1} J_{i+g_{t}+1}\right)^{2} K_{k+g_{t}+1}$.

- Step 4: $\mathcal{L}\left(S_{\infty}\right)$ is the unique solution of (6.10). 
The proof of Steps 1-4 is found in the supplementary material [30]. These results can be obtained from Vervaat's Theorem 1.5 in [40] where discrete times are replaced by the renewal time $g_{t}$. Given Steps 1-4, the proof of (6.10) is completed as follows.

Notice that

$$
S_{\infty}-S_{g_{t}}=J_{1}^{2} J_{2}^{2} \cdots J_{g_{t}}^{2} K_{g_{t}+1}+J_{1}^{2} J_{2}^{2} \cdots J_{g_{t}+1}^{2}\left(\sum_{k=1}^{\infty}\left(\prod_{i=1}^{k-1} J_{i+g_{t}+1}\right)^{2} K_{k+g_{t}+1}\right) .
$$

We will prove that the right-hand side in (6.18) converges to zero in probability as $t \rightarrow \infty$. Since by Step $1, \mathcal{L}\left(\widetilde{S}_{g_{t}}\right)=\mathcal{L}\left(S_{g_{t}}\right)$ holds. Then the assertion $\tilde{S}_{g_{t}} \stackrel{d}{\rightarrow} S_{\infty}$ will follow immediately. The first term on the right-hand side in (6.18) may be written as

$$
J_{1}^{2} J_{2}^{2} \cdots J_{g_{t}}^{2} K_{g_{t}+1}=\exp \left\{t\left(\frac{2 \sum_{i=1}^{g_{t}} \log J_{i}}{g_{t}}+\frac{\log K_{g_{t}+1}}{g_{t}}\right) \frac{g_{t}}{t}\right\} .
$$

By the renewal theorem,

$$
\frac{g_{t}}{t} \stackrel{\text { a.s. }}{\rightarrow} \frac{1}{E\left|I_{1}\right|} \quad \text { as } t \rightarrow \infty
$$

and by Assumption 2(a), as $t \rightarrow \infty$,

$$
\frac{\sum_{i=1}^{g_{t}} \log J_{i}}{g_{t}} \stackrel{\text { a.s. }}{\rightarrow} E \log J_{1}=-E \int_{\tau_{0}}^{\tau_{1}} a\left(X_{s}\right) d s=-E\left|I_{1}\right| E_{\pi} a(\cdot)<0 .
$$

Together with Step 2, we have therefore

$$
\left(\frac{2 \sum_{i=1}^{g_{t}} \log J_{i}}{g_{t}}+\frac{\log K_{g_{t}+1}}{g_{t}}\right) \frac{g_{t}}{t} \stackrel{\text { a.s. }}{\rightarrow}-2 E_{\pi} a(\cdot)<0 \quad \text { as } t \rightarrow \infty
$$

from which $J_{1}^{2} J_{2}^{2} \cdots J_{g_{t}}^{2} K_{g_{t}+1} \stackrel{\text { a.s. }}{\rightarrow} 0$ as $t \rightarrow \infty$ follows. For the second term on the right-hand side in (6.18), by Step 3,

$$
\mathcal{L}\left(\sum_{k=1}^{\infty}\left(\prod_{i=1}^{k-1} J_{i+g_{t}+1}\right)^{2} K_{k+g_{t}+1}\right)=\mathcal{L}\left(S_{\infty}\right)
$$

and

$$
J_{1}^{2} J_{2}^{2} \cdots J_{g_{t}+1}^{2}=\exp \left\{t\left(\frac{2 \sum_{i=1}^{g_{t}+1} \log J_{i}}{g_{t}+1}\right) \frac{g_{t}+1}{t}\right\} \stackrel{\text { a.s. }}{\rightarrow} 0 \quad \text { as } t \rightarrow \infty,
$$

from which Slutsky's theorem gives

$$
J_{1}^{2} J_{2}^{2} \ldots J_{g_{t}+1}^{2}\left(\sum_{k=1}^{\infty}\left(\prod_{i=1}^{k-1} J_{i+g_{t}+1}\right)^{2} K_{k+g_{t}+1}\right) \stackrel{P}{\rightarrow} 0 \quad \text { as } t \rightarrow \infty .
$$

Given Steps 1-4 we have thus shown (6.10). 
Given the proof of (6.17) in the supplementary material [30], the proof Lemma 6.2 is complete.

Remark 6.1. We acknowledge that Lemma 6.2 can be shown using Anscombe's condition (page 16 in Gut [23]) for $g_{t} \stackrel{P}{\rightarrow} \infty$ as $t \rightarrow \infty$, together with

$$
R_{\tau_{n}}:=\left(\left(\prod_{k=1}^{n} J_{k}^{j}\right) J_{0}^{j},\left(\prod_{k=1}^{n} J_{k}^{j}\right)^{2} K_{0}^{j}+\sum_{k=1}^{n}\left(\prod_{l=k+1}^{n} J_{l}^{j}\right)^{2} K_{k}^{j}\right) \stackrel{d}{\rightarrow}\left(0, V_{j}^{*}\right) \quad \text { as } n \rightarrow \infty .
$$

Verification of the above convergence and Anscombe's condition for $\left(R_{\tau_{n}}\right)_{n \geq 1}$ lead to arguments similar to Steps 1-4 above.

\subsection{Proof of Lemma 6.3}

Proof. The main assertion will follow if we prove that, for any $A$ with $P\left[H_{\infty} \in \partial A\right]=0$,

$$
\lim _{t \rightarrow \infty} P\left[H_{t} \in A, X_{t}=j\right]=\pi_{j} \lim _{t \rightarrow \infty} P\left[H_{t} \in A\right]=\pi_{j} P\left[H_{\infty} \in A\right] .
$$

Then, by Slutsky's theorem,

$$
\lim _{t \rightarrow \infty} P\left[L_{t}^{(1)}+L_{t}^{(2)} H_{t} \in A, X_{t}=j\right]=\lim _{t \rightarrow \infty} P\left[H_{t} \in A, X_{t}=j\right] .
$$

We prove (6.19) in the following two steps:

- Step 1: $\lim _{t \rightarrow \infty} P\left[H_{t-\varepsilon(t)} \in A \mid X_{t}=j\right]=P\left[H_{\infty} \in A\right]$.

- Step 2: $\lim _{t \rightarrow \infty} P\left[H_{t} \in A \mid X_{t}=j\right]=\lim _{t \rightarrow \infty} P\left[H_{t-\varepsilon(t)} \in A \mid X_{t}=j\right]$.

Step 1: Notice that

$$
\begin{aligned}
P & {\left[H_{t-\varepsilon(t)} \in A \mid X_{t}=j\right] } \\
& =\sum_{j^{\prime} \in S} P\left[H_{t-\varepsilon(t)} \in A \mid X_{t-\varepsilon(t)}=j^{\prime}, X_{t}=j\right] \frac{P\left[X_{t-\varepsilon(t)}=j^{\prime}, X_{t}=j\right]}{P\left[X_{t}=j\right]} \\
& =\sum_{j^{\prime} \in S} P\left[H_{t-\varepsilon(t)} \in A \mid X_{t-\varepsilon(t)}=j^{\prime}\right] \frac{P\left[X_{t}=j \mid X_{t-\varepsilon(t)}=j^{\prime}\right] P\left[X_{t-\varepsilon(t)}=j^{\prime}\right]}{P\left[X_{t}=j\right]} \\
& =\sum_{j^{\prime} \in S} P\left[H_{t-\varepsilon(t)} \in A, X_{t-\varepsilon(t)}=j^{\prime}\right] \frac{P_{j^{\prime} j}(0, \varepsilon(t))}{P\left[X_{t}=j\right]} \\
& =P\left[H_{t-\varepsilon(t)} \in A\right]+\sum_{j^{\prime} \in S} P\left[H_{t-\varepsilon(t)} \in A, X_{t-\varepsilon(t)}=j^{\prime}\right]\left(\frac{P_{j^{\prime} j}(0, \varepsilon(t))}{P\left[X_{t}=j\right]}-1\right) .
\end{aligned}
$$

The second equality follows by observing $H_{t-\varepsilon(t)}$ is conditionally independent of $X_{t}$ given $X_{t-\varepsilon(t)}$. The third equality follows since the Markov chain $X$ is time homogenous. Observe that 
irreducibility implies that $\pi_{j}>0$ for all $j \in S$. By ergodicity of $X, \lim _{t \rightarrow \infty} P_{j^{\prime} j}(0, \varepsilon(t)) / P\left[X_{t}=\right.$ $j]=1$ since both the numerator and denominator converge to $\pi_{j}$. For a fixed $j \in S$ and any $\delta \in\left(0, \pi_{j}\right)$, there exists $t_{\delta}>0$ such that $\left|P\left[X_{t}=j\right]-\pi_{j}\right| \leq \delta$ for $t \geq t_{\delta}$. Notice that each term in the sum (6.20) converges to 0 as $t \rightarrow \infty$. Notice also that, for $t \geq t_{\delta}$,

$$
\begin{aligned}
P\left[H_{t-\varepsilon(t)} \in A, X_{t-\varepsilon(t)}=j^{\prime}\right]\left|\frac{P_{j^{\prime} j}(0, \varepsilon(t))}{P\left[X_{t}=j\right]}-1\right| & \leq P\left[X_{t-\varepsilon(t)}=j^{\prime}\right] \frac{2}{P\left[X_{t}=j\right]} \\
& \leq P\left[X_{t-\varepsilon(t)}=j^{\prime}\right] \frac{2}{\pi_{j}-\delta}
\end{aligned}
$$

and that the upper bound is summable over $j^{\prime} \in S$ and has a limit as $t \rightarrow \infty$ which is also summable over $j^{\prime} \in S$. Hence, by Pratt's lemma (page 101 in Schilling [34]), the sum in (6.20) converges to 0 as $t \rightarrow \infty$. Since $H_{t-\varepsilon(t)} \stackrel{d}{\rightarrow} H_{\infty}$ as $t \rightarrow \infty$ the proof of Step 1 is complete.

Step 2: Take any $\delta>0$ and define $A^{\delta}:=\{x: d(x, A)<\delta\}, A^{-\delta}:=\left\{x \in A: d\left(x, A^{c}\right)>\delta\right\}$ and note that $A^{-\delta} \subseteq A \subseteq A^{\delta}$. Denoting $B_{t}:=H_{t}-H_{t-\varepsilon(t)}$ one has

$$
P\left[H_{t} \in A \mid X_{t}=j\right]=P\left[H_{t-\varepsilon(t)}+B_{t} \in A,\left|B_{t}\right| \leq \delta \mid X_{t}=j\right]+C_{t},
$$

where

$$
\limsup _{t \rightarrow \infty} C_{t} \leq \limsup _{t \rightarrow \infty} \frac{P\left[\left|B_{t}\right|>\delta\right]}{P\left[X_{t}=j\right]}=0 .
$$

The first term on the right-hand side in (6.21) satisfies

$$
\begin{aligned}
P\left[H_{t-\varepsilon(t)} \in A^{-\delta} \mid X_{t}=j\right] & \leq P\left[H_{t-\varepsilon(t)}+B_{t} \in A,\left|B_{t}\right| \leq \delta \mid X_{t}=j\right] \\
& \leq P\left[H_{t-\varepsilon(t)} \in A^{\delta} \mid X_{t}=j\right] .
\end{aligned}
$$

The assertion follows from Step 1 together with letting $\delta \rightarrow 0$.

\subsection{Proof of Theorem 2(a)}

Proof. Set $\widetilde{Y}_{t}:=Y_{0}+\int_{0}^{t} b\left(X_{S}\right) \boldsymbol{\Phi}^{-1}(0, s) d W_{s}$ and notice that $Y_{t}=\boldsymbol{\Phi}(0, t) \widetilde{Y}_{t}$. Fix an arbitrary $j \in S$ and set

$$
C_{t}^{(1)}:=\frac{\left(\prod_{l=1}^{g_{t}^{j}} J_{l}^{j}\right)^{1 / \sqrt{t}}}{e^{-\sqrt{t} E_{\pi} a(\cdot)}}, \quad C_{t}^{(2)}:=\left(e^{-a(j)\left(t-\tau^{j}{ }_{g}^{j}\right)} J_{0}^{j}\right)^{1 / \sqrt{t}}
$$

and

$$
C_{t}^{(3)}:=\left|y_{0}+\left(\frac{G_{j}^{2 a, b^{2}}\left(t-\tau_{g_{t}^{j}}^{j}\right)}{\boldsymbol{\Phi}^{2}(0, t)}+\frac{K_{0}^{j}+S_{g_{t}}^{*}}{\left(J_{0}^{j}\right)^{2}}\right)^{1 / 2} N\right|^{1 / \sqrt{t}},
$$


where $N \sim \mathrm{N}(0,1), N \Perp X$, and

$$
S_{n}^{*}:=\sum_{i=1}^{n} \frac{K_{i}^{j}}{\left(J_{i}^{j}\right)^{2}} \prod_{k=1}^{i-1}\left(\frac{1}{J_{k}^{j}}\right)^{2}, \quad n \geq 1 .
$$

Notice that

$$
\begin{aligned}
& \mathcal{L}\left(\left|\widetilde{Y}_{t}\right|^{1 / \sqrt{t}} \mid X_{0}=i, X_{t}=j, Y_{0}=y_{0}\right) \\
& \quad=\mathcal{L}\left(\left|y_{0}+\left(\boldsymbol{\Phi}^{-2}(0, t) \int_{0}^{t} b^{2}\left(X_{s}\right) \boldsymbol{\Phi}^{2}(s, t) d s\right)^{1 / 2} N\right|^{1 / \sqrt{t}} \mid X_{0}=i, X_{t}=j, Y_{0}=y_{0}\right) \\
& \quad=\mathcal{L}\left(C_{t}^{(3)} \mid X_{0}=i, X_{t}=j, Y_{0}=y_{0}\right)
\end{aligned}
$$

and

$$
\begin{aligned}
& \mathcal{L}\left(\frac{\left|Y_{t}\right|^{1 / \sqrt{t}}}{e^{-\sqrt{t} E_{\pi} a(\cdot)}} \mid X_{0}=i, X_{t}=j, Y_{0}=y_{0}\right) \\
& \quad=\mathcal{L}\left(C_{t}^{(1)} C_{t}^{(2)} C_{t}^{(3)} \mid X_{0}=i, X_{t}=j, Y_{0}=y_{0}\right) .
\end{aligned}
$$

For any $A \in \mathcal{B}(\mathbb{R})$,

$$
\begin{aligned}
& P\left[\frac{\left|Y_{t}\right|^{1 / \sqrt{t}}}{e^{-\sqrt{t} E_{\pi} a(\cdot)}} \in A \mid X_{0}=i, Y_{0}=y_{0}\right] \\
& \quad=\sum_{j \in S} P\left[C_{t}^{(1)} C_{t}^{(2)} C_{t}^{(3)} \in A, X_{t}=j \mid X_{0}=i, Y_{0}=y_{0}\right] \\
& \quad=\sum_{j \in S} P_{i j}(0, t) P\left[C_{t}^{(1)} C_{t}^{(2)} C_{t}^{(3)} \in A \mid X_{0}=i, X_{t}=j, Y_{0}=y_{0}\right] .
\end{aligned}
$$

By the ergodic theorem and the renewal theorem, $E \log J_{1}^{j}=-E\left|I_{1}^{j}\right| E_{\pi} a(\cdot), g_{t}^{j} / t \stackrel{\text { a.s. }}{\rightarrow} 1 / E\left|I_{1}^{j}\right|$ as $t \rightarrow \infty$. Therefore, by applying the central limit theorem for the renewal reward process (see, e.g., Theorem 2.2.5 in Tijms [39]), when the reward is $\log J_{l}^{j}$ for the $l$-th interval $I_{l}^{j}$, together with the continuous mapping theorem using the map $x \mapsto e^{x}$, with $N \sim \mathrm{N}(0,1)$,

$$
\mathcal{L}\left(C_{t}^{(1)} \mid X_{0}=i, Y_{0}=y_{0}\right) \stackrel{w}{\rightarrow} \mathcal{L}\left(\exp \left\{\frac{\sigma_{j}}{\sqrt{E\left|I_{1}^{j}\right|}} N\right\}\right) \quad \text { as } t \rightarrow \infty .
$$

Applying arguments similar to those in Step 4 in the proof of Lemma 6.2, using

$$
E \log \frac{1}{\left(J_{1}^{j}\right)^{2}}<0, \quad E \log ^{+} \frac{K_{1}^{j}}{\left(J_{1}^{j}\right)^{2}}<\infty
$$


we find that

$$
S_{g_{t}}^{*} \stackrel{d}{\rightarrow} S^{*} \quad \text { as } t \rightarrow \infty, \quad S^{*} \stackrel{d}{=} \frac{1}{\left(J_{1}^{j}\right)^{2}} S^{*}+\frac{K_{1}^{j}}{\left(J_{1}^{j}\right)^{2}}, \quad S^{*} \Perp\left(\frac{1}{J_{1}^{j}}, \frac{K_{1}^{j}}{\left(J_{1}^{j}\right)^{2}}\right) .
$$

In the transient regime,

$$
\frac{\log \left|\Phi^{2}(0, t)\right|}{t} \stackrel{\text { a.s. }}{\rightarrow}-2 E_{\pi} a(\cdot)>0 \quad \text { as } t \rightarrow \infty,
$$

so $\boldsymbol{\Phi}(0, t)$ diverges exponentially fast as $t \rightarrow \infty$. Moreover, $G_{j}^{2 a, b^{2}}\left(t-\tau_{g_{t}}^{j}\right)$ is $O_{P}(1)$. Using that $S_{g_{t}}^{*} \stackrel{d}{\rightarrow} S^{*}$ as $t \rightarrow \infty$ now leads to

$$
\mathcal{L}\left(\left(C_{t}^{(3)}\right)^{\sqrt{t}} \mid X_{0}=i, Y_{0}=y_{0}\right) \stackrel{w}{\rightarrow} P\left[\left|y_{0}+\left(\frac{K_{0}^{j}+S^{*}}{\left(J_{0}^{j}\right)^{2}}\right)^{1 / 2} N\right| \in \cdot\right] \quad \text { as } t \rightarrow \infty
$$

which implies that

$$
\mathcal{L}\left(C_{t}^{(3)} \mid X_{0}=i, Y_{0}=y_{0}\right) \stackrel{w}{\rightarrow} \delta_{1} \quad \text { as } t \rightarrow \infty
$$

Moreover, since both $t-\tau_{g_{t}^{j}}^{j}$ and $J_{0}^{j}$ are $O_{P}(1)$,

$$
\mathcal{L}\left(C_{t}^{(2)} \mid X_{0}=i, Y_{0}=y_{0}\right) \stackrel{w}{\rightarrow} \delta_{1} \quad \text { as } t \rightarrow \infty
$$

Therefore combining (6.23), (6.25) and (6.26), and applying Lemma 6.3, taking

$$
L_{t}^{(1)}:=0, \quad L_{t}^{(2)}:=C_{t}^{(2)} C_{t}^{(3)}, \quad H_{t}:=C_{t}^{(1)}
$$

in the statement of Lemma 6.3, show that

$$
\mathcal{L}\left(C_{t}^{(1)} C_{t}^{(2)} C_{t}^{(3)} \mid X_{0}=i, X_{t}=j, Y_{0}=y_{0}\right) \stackrel{w}{\rightarrow} \mathcal{L}\left(\exp \left\{\frac{\sigma_{j}}{\sqrt{E \mid I_{1}^{j}} \mid} N\right\}\right) \quad \text { as } t \rightarrow \infty
$$

given that $C_{t}^{(1)}$ satisfies condition (6.11) imposed on $H_{t}$ in Lemma 6.3. $C_{t}^{(1)}$ satisfies condition (6.11) if $M_{t}:=\log C_{t}^{(1)}$ satisfies (6.11), that is, for some increasing function $t \mapsto \varepsilon(t)$ such that $\varepsilon(t) \rightarrow \infty$ and $\varepsilon(t) / t \rightarrow 0$ as $t \rightarrow \infty$,

$$
M_{t}-M_{t-\varepsilon(t)} \stackrel{P}{\rightarrow} 0 \quad \text { as } t \rightarrow \infty
$$


Lemma 6.5 below verifies this condition. Finally, since $\lim _{t \rightarrow \infty} P_{i j}(0, t)=\pi_{j}$, the dominated convergence theorem applied to

$$
\begin{aligned}
& P\left[\frac{\left|Y_{t}\right|^{1 / \sqrt{t}}}{e^{-\sqrt{t} E_{\pi} a(\cdot)}} \in A \mid X_{0}=i, Y_{0}=y_{0}\right] \\
& \quad=\sum_{j \in S} P_{i j}(0, t) P\left[C_{t}^{(1)} C_{t}^{(2)} C_{t}^{(3)} \in A \mid X_{0}=i, X_{t}=j, Y_{0}=y_{0}\right]
\end{aligned}
$$

using the trivial upper bound 1 for the second factor of terms in the above sum, completes the proof.

Lemma 6.5. There exists an increasing function $t \mapsto \varepsilon(t)$ such that $\varepsilon(t) \rightarrow \infty$ and $\varepsilon(t) / t \rightarrow 0$ as $t \rightarrow \infty$ such that (6.27) holds.

The proof of Lemma 6.5 is found in the supplementary material [30].

\subsection{Proof of Theorem 2(b)}

Before proving Theorem 2(b) we introduce some notations useful for random walk related arguments. Fix two arbitrary states $i, j \in S$. Given $E_{\pi} a(\cdot)=0$, the random walk $\left(S_{n}^{\otimes j}\right)_{n \geq 0}$ given by $S_{0}^{\otimes j}:=0$ and $S_{n}^{\otimes j}:=\sum_{k=1}^{n} \log J_{k}^{j}$ for $n \geq 1$ is null-recurrent. Recall that $\log J_{k}^{j}=$ $-\int_{\tau_{k-1}^{j}}^{\tau_{k}^{j}} a\left(X_{S}\right) d s$ with mean $-E_{\pi} a(\cdot) E\left|I_{1}^{j}\right|=0$ and variance $\sigma_{j}^{2}$. For this random walk we define the sequence $\left(Z_{k}^{\otimes j}\right)_{k \geq 1}$ of ladder variables as follows. Define the sequence $\left(T_{k}^{\otimes j}\right)_{k \geq 1}$ such that

$$
T_{1}^{\otimes j}:=\inf \left\{m: S_{m}^{\otimes}>0\right\}, \quad T_{k}^{\otimes j}:=\inf \left\{m>T_{k-1}^{\otimes j}: S_{m}^{\otimes j}>S_{T_{k-1}}^{\otimes j}\right\} .
$$

The ladder variables are defined as $Z_{k}^{\otimes j}:=S_{T_{k}^{\otimes j}}^{\otimes j}-S_{T_{k-1}^{\otimes j}}^{\otimes j}$. Let $M_{n}^{\otimes j}:=\max _{1 \leq k \leq n} \sum_{i=1}^{k} \log J_{i}^{j}$.

Proof. We begin by expanding (6.12) differently from what was done in the proof of Theorem 2(a). Since $E_{\pi} a(\cdot)=0$, instead of extracting $\boldsymbol{\Phi}(0, t)$ as in the proof of Theorem 2(a), we will extract $Z_{n}^{*}:=\max _{1 \leq k \leq n}\left(\prod_{i=1}^{k-1} J_{i}^{j}\right)^{2} K_{k}^{j}$, for $n=g_{t}^{j}$. Note that all $J_{k}^{j}, K_{k}^{j}$ are non-negative. Let $N \sim \mathrm{N}(0,1)$ and recall notations $Q_{t}^{(1)}$ and $Q_{t}^{(2)}$ in (6.13) and $S_{n}:=\sum_{k=1}^{n}\left(\prod_{i=1}^{k-1} J_{i}^{j}\right)^{2} K_{k}^{j}$. Using that $\widetilde{S}_{g_{t}^{j}} \stackrel{d}{=} S_{g_{t}^{j}}$ for all $t>0$ (Step 1 in the proof of (6.10)), on $\left\{X_{0}=i, X_{t}=j\right\}$,

$$
\begin{aligned}
Y_{t} & \stackrel{d}{=} Y_{0} Q_{t}^{(1)}+\sqrt{Q_{t}^{(2)}} N \\
& =Y_{0} e^{-a(j)\left(t-\tau^{j}{ }^{j}\right)}\left(\prod_{k=1}^{g_{t}^{j}} J_{k}^{j}\right) J_{0}^{j}
\end{aligned}
$$




$$
\begin{aligned}
& +\left(G_{j}^{2 a, b^{2}}\left(t-\tau_{g_{t}^{j}}^{j}\right)+e^{-2 a(j)\left(t-\tau^{j}{ }^{j}\right)}\left[\left(\prod_{k=1}^{g_{t}^{j}} J_{k}^{j}\right)^{2} K_{0}^{j}+\widetilde{S}_{g_{t}^{j}}\right]\right)^{1 / 2} N \\
& \stackrel{d}{=} \sqrt{Z_{g_{t}^{j}}^{*}}\left(Y_{0} B_{t}^{(1)}+\left(B_{t}^{(2)}+B_{t}^{(3)} \frac{S_{g_{t}^{j}}}{Z_{g_{t}^{j}}^{*}}\right)^{1 / 2} N\right),
\end{aligned}
$$

where

$$
\begin{aligned}
& B_{t}^{(1)}:=\frac{1}{\sqrt{Z_{g_{t}^{*}}{ }^{j}}} e^{-a(j)\left(t-\tau^{j}{ }^{j}\right)}\left(\prod_{k=1}^{g_{t}^{j}} J_{k}^{j}\right) J_{0}^{j}, \\
& B_{t}^{(2)}:=\frac{1}{Z_{g_{t}}^{*}}\left(G_{j}^{2 a, b^{2}}\left(t-\tau_{g_{t}^{j}}^{j}\right)+e^{-2 a(j)\left(t-\tau^{j}{ }^{j}\right)}\left(\prod_{k=1}^{g_{t}^{j}} J_{k}^{j}\right)^{2} K_{0}^{j}\right), \\
& B_{t}^{(3)}:=e^{-2 a(j)\left(t-\tau^{j}{ }^{j}\right)} .
\end{aligned}
$$

Denote the second factor in the product (6.28) by $B_{t}$. Since $K_{n}^{j}\left(\prod_{i=1}^{n-1} J_{i}^{j}\right)^{2} \leq Z_{n}^{*}$ for all $n \geq 1$, $B_{t}^{(1)}, B_{t}^{(2)}, B_{t}^{(3)}$ are all $O_{P}(1)$. Since $Z_{n}^{*} \leq S_{n} \leq n Z_{n}^{*}$ for all $n \geq 1,\left|B_{t}\right|=O_{P}(\sqrt{t})$ and is strictly positive. Moreover,

$$
\left|B_{t}\right|^{1 / \sqrt{t}}=\exp \left\{\frac{1}{\sqrt{t}} \log \left|Y_{0} B_{t}^{(1)}+\left(B_{t}^{(2)}+B_{t}^{(3)} \frac{S_{g_{t}^{j}}}{Z_{g_{t}^{j}}^{*}}\right)^{1 / 2} N\right|\right\} \stackrel{P}{\rightarrow} 1 \quad \text { as } t \rightarrow \infty .
$$

Consider the probability

$$
\begin{aligned}
P & {\left[\left|Y_{t}\right|^{1 / \sqrt{t}} \in A \mid X_{0}=i, Y_{0}=y_{0}\right] } \\
& =\sum_{j \in S} P_{i j}(0, t) P\left[\left(Z_{g_{t}^{j}}^{*}\right)^{\frac{1}{2 \sqrt{t}}}\left|B_{t}\right|^{\frac{1}{\sqrt{t}}} \in A \mid X_{0}=i, X_{t}=j, Y_{0}=y_{0}\right] .
\end{aligned}
$$

We want to let $t \rightarrow \infty$ in (6.29) and in order to get rid of conditioning on $X_{t}=j$ on the righthand side we apply Lemma 6.3 with

$$
\left(L_{t}^{(1)}, L_{t}^{(2)}, H_{t}\right):=\left(0,\left|B_{t}\right|^{1 / \sqrt{t}},\left(Z_{g_{t}^{j}}^{*}\right)^{1 /(2 \sqrt{t})}\right) .
$$

This leads to

$$
\begin{aligned}
& \lim _{t \rightarrow \infty} P\left[\left|Y_{t}\right|^{1 / \sqrt{t}} \in A \mid X_{0}=i, Y_{0}=y_{0}\right] \\
& \quad=\sum_{j \in S} \pi_{j} \lim _{t \rightarrow \infty} P\left[\left(Z_{g_{t}^{*}}^{*}\right)^{1 /(2 \sqrt{t})} \in A \mid X_{0}=i\right]
\end{aligned}
$$


if the right-hand limit in (6.30) exists and $\left(\log Z_{g_{t}}^{*}\right) / \sqrt{t}$ satisfies condition (6.27) in the role of $M_{t}$. Lemma 6.6 below verifies that. Immediately from the definitions of $M_{n}^{\otimes j}$ and $Z_{n}^{*}$ (see also (4.4) in Hitczenko and Wesolowski [26]) follow

$$
2 M_{g_{t}^{j}}^{\otimes j}-\max _{1 \leq k \leq g_{t}^{j}} \log K_{k}^{j} \leq \log Z_{g_{t}^{j}}^{*} \leq 2 M_{g_{t}^{j}}^{\otimes j}+\max _{1 \leq k \leq g_{t}^{j}} \log K_{k}^{j} .
$$

It is shown in [26] that

$$
\frac{M_{n}^{\otimes j}}{\sqrt{n}} \stackrel{d}{\rightarrow} \sigma_{j}|N| \quad \text { and } \quad \frac{\max _{1 \leq k \leq n} \log K_{k}^{j}}{\sqrt{n}} \stackrel{P}{\rightarrow} 0 \quad \text { as } n \rightarrow \infty .
$$

Lemma 6.6 below gives the renewal time version of the above convergence. In particular,

$$
\frac{\log Z_{g_{t}}^{*}}{2 \sqrt{t}} \stackrel{d}{\rightarrow} \sigma_{j}|N| \quad \text { as } t \rightarrow \infty
$$

which concludes the proof of Theorem 2(b).

Lemma 6.6. Under the assumptions of Theorem 2(b), as $t \rightarrow \infty$,
(a) $\frac{\max _{1 \leq k \leq g_{t}^{j}} \log K_{g_{t}^{j}}^{j}}{\sqrt{g_{t}^{j}}} \stackrel{\text { a.s. }}{\rightarrow} 0$,
(b) $\frac{M_{g_{t}^{j}}^{\otimes j}}{\sqrt{g_{t}^{j}}} \stackrel{d}{\rightarrow} \sigma_{j}|N|$,
(c) $\frac{\log Z_{g_{t}^{j}}^{*}}{\sqrt{t}}$ satisfies (6.27) in the role of $M_{t}$.

The proof of Lemma 6.6 is found in the supplementary material [30].

\section{Acknowledgements}

The authors thank the two anonymous reviewers for comments and suggestions that significantly improved the paper. The second author thanks Daniele Cappelletti and Carsten Wiuf for several illuminating discussions on a related problem in Cappelletti et al. [11].

\section{Supplementary Material}

Supplement: Proofs of some of the results (DOI: 10.3150/20-BEJ1196SUPP; .pdf). The supplementary material contains the proofs of Propositions 3.7, 5.1 and 5.2, proof of a part of Lemma 6.2, proofs of Lemmas 6.4, 6.5 and 6.6, and additional details for Remark 3.3. 


\section{References}

[1] Abourashchi, N., Clacher, I., Freeman, M.C., Hillier, D., Kemp, M. and Zhang, Q. (2016). Pension plan solvency and extreme market movements: A regime switching approach. Eur. J. Finance 22 $1292-1319$.

[2] Ang, A. and Timmermann, A. (2012). Regime changes and financial markets. Annu. Rev. Financ. Econ. 4 313-337.

[3] Bardet, J.-B., Guérin, H. and Malrieu, F. (2010). Long time behavior of diffusions with Markov switching. ALEA Lat. Am. J. Probab. Math. Stat. 7 151-170. MR2653702

[4] Basak, G.K., Bisi, A. and Ghosh, M.K. (1996). Stability of a random diffusion with linear drift. J. Math. Anal. Appl. 202 604-622. MR1406250 https://doi.org/10.1006/jmaa.1996.0336

[5] Behme, A. and Lindner, A. (2015). On exponential functionals of Lévy processes. J. Theoret. Probab. 28 681-720. MR3370671 https://doi.org/10.1007/s10959-013-0507-y

[6] Benaïm, M. and Lobry, C. (2016). Lotka-Volterra with randomly fluctuating environments or "How switching between beneficial environments can make survival harder". Ann. Appl. Probab. 26 37543785. MR3582817 https://doi.org/10.1214/16-AAP1192

[7] BenSaida, A. (2015). The frequency of regime switching in financial market volatility. J. Empir. Finance 32 63-79.

[8] Bertoin, J. and Yor, M. (2005). Exponential functionals of Lévy processes. Probab. Surv. 2 191-212. MR2178044 https://doi.org/10.1214/154957805100000122

[9] Buraczewski, D., Damek, E. and Mikosch, T. (2016). Stochastic Models with Power-Law Tails. Springer Series in Operations Research and Financial Engineering. Cham: Springer. MR3497380 https://doi.org/10.1007/978-3-319-29679-1

[10] Buraczewski, D. and Iksanov, A. (2015). Functional limit theorems for divergent perpetuities in the contractive case. Electron. Commun. Probab. 20 no. 10, 14. MR3314645 https://doi.org/10.1214/ECP. v20-3915

[11] Cappelletti, D., Pal Majumder, A. and Wiuf, C. (2019). Long time asymptotics of stochastic reaction systems. Preprint. Available at arXiv:1912.00401.

[12] Cloez, B. and Hairer, M. (2015). Exponential ergodicity for Markov processes with random switching. Bernoulli 21 505-536. MR3322329 https://doi.org/10.3150/13-BEJ577

[13] Cox, J.C., Ingersoll, J.E. Jr. and Ross, S.A. (1985). A theory of the term structure of interest rates. Econometrica 53 385-407. MR0785475 https://doi.org/10.2307/1911242

[14] de Saporta, B. and Yao, J.-F. (2005). Tail of a linear diffusion with Markov switching. Ann. Appl. Probab. 15 992-1018. MR2114998 https://doi.org/10.1214/105051604000000828

[15] Djehiche, B. and Löfdahl, B. (2018). A hidden Markov approach to disability insurance. N. Am. Actuar. J. 22 119-136. MR3774616 https://doi.org/10.1080/10920277.2017.1387570

[16] Erdös, P. and Kac, M. (1946). On certain limit theorems of the theory of probability. Bull. Amer. Math. Soc. 52 292-302. MR0015705 https://doi.org/10.1090/S0002-9904-1946-08560-2

[17] Feng, R., Kuznetsov, A. and Yang, F. (2019). Exponential functionals of Lévy processes and variable annuity guaranteed benefits. Stochastic Process. Appl. 129 604-625. MR3907011 https://doi.org/10. 1016/j.spa.2018.03.011

[18] Fink, H., Klimova, Y., Czado, C. and Stöber, J. (2017). Regime switching vine copula models for global equity and volatility indices. Econometrics 5 3-17.

[19] Gao, H., Mamon, R., Liu, X. and Tenyakov, A. (2015). Mortality modelling with regime-switching for the valuation of a guaranteed annuity option. Insurance Math. Econom. 63 108-120. MR3372586 https://doi.org/10.1016/j.insmatheco.2015.03.018

[20] Genon-Catalot, V., Jeantheau, T. and Larédo, C. (2000). Stochastic volatility models as hidden Markov models and statistical applications. Bernoulli 6 1051-1079. MR1809735 https://doi.org/10.2307/ 3318471 
[21] Gjessing, H.K. and Paulsen, J. (1997). Present value distributions with applications to ruin theory and stochastic equations. Stochastic Process. Appl. 71 123-144. MR1480643 https://doi.org/10.1016/ S0304-4149(97)00072-0

[22] Gray, A., Greenhalgh, D., Mao, X. and Pan, J. (2012). The SIS epidemic model with Markovian switching. J. Math. Anal. Appl. 394 496-516. MR2927473 https://doi.org/10.1016/j.jmaa.2012.05.029

[23] Gut, A. (2009). Stopped Random Walks: Limit Theorems and Applications, 2nd ed. Springer Series in Operations Research and Financial Engineering. New York: Springer. MR2489436 https://doi.org/10. 1007/978-0-387-87835-5

[24] Hairer, M. (2010). Convergence of Markov processes. Lecture notes.

[25] Hardy, M.R. (2001). A regime-switching model of long-term stock returns. N. Am. Actuar. J. 5 41-53. MR1988438 https://doi.org/10.1080/10920277.2001.10595984

[26] Hitczenko, P. and Wesołowski, J. (2011). Renorming divergent perpetuities. Bernoulli 17 880-894. MR2817609 https://doi.org/10.3150/10-BEJ297

[27] Hou, T. and Shao, J. (2019). Heavy tail and light tail of Cox-Ingersoll-Ross processes with regimeswitching. Sci. China Math.. https://doi.org/10.1007/s11425-017-9392-5.

[28] Jeanblanc, M., Yor, M. and Chesney, M. (2009). Mathematical Methods for Financial Markets. Springer Finance. London: Springer London, Ltd. MR2568861 https://doi.org/10.1007/ 978-1-84628-737-4

[29] Lin, X.S., Tan, K.S. and Yang, H. (2009). Pricing annuity guarantees under a regime-switching model. N. Am. Actuar. J. 13 316-338. MR2579569 https://doi.org/10.1080/10920277.2009.10597557

[30] Lindskog, F. and Pal Majumder, A. (2020). Supplement to "Exact long time behavior of some regime switching stochastic processes." https://doi.org/10.3150/20-BEJ1196SUPP

[31] Mao, X. and Yuan, C. (2006). Stochastic Differential Equations with Markovian Switching. London: Imperial College Press. MR2256095 https://doi.org/10.1142/p473

[32] Maulik, K. and Zwart, B. (2006). Tail asymptotics for exponential functionals of Lévy processes. Stochastic Process. Appl. 116 156-177. MR2197972 https://doi.org/10.1016/j.spa.2005.09.002

[33] Norris, J.R. (1998). Markov Chains. Cambridge Series in Statistical and Probabilistic Mathematics 2. Cambridge: Cambridge Univ. Press. MR1600720

[34] Schilling, R.L. (2005). Measures, Integrals and Martingales. New York: Cambridge Univ. Press. MR2200059 https://doi.org/10.1017/CBO9780511810886

[35] Shao, J. (2014). Ergodicity of one-dimensional regime-switching diffusion processes. Sci. China Math. 57 2407-2414. MR3266501 https://doi.org/10.1007/s11425-014-4853-8

[36] Shao, J. (2015). Ergodicity of regime-switching diffusions in Wasserstein distances. Stochastic Process. Appl. 125 739-758. MR3293301 https://doi.org/10.1016/j.spa.2014.10.007

[37] Shao, J. (2015). Criteria for transience and recurrence of regime-switching diffusion processes. Electron. J. Probab. 20 no. 63, 15. MR3361251 https://doi.org/10.1214/EJP.v20-4018

[38] Shen, Y. and Siu, T.K. (2013). Longevity bond pricing under stochastic interest rate and mortality with regime-switching. Insurance Math. Econom. 52 114-123. MR3023660 https://doi.org/10.1016/ j.insmatheco.2012.11.006

[39] Tijms, H.C. (2003). A First Course in Stochastic Models. Chichester: Wiley. MR2190630 https://doi.org/10.1002/047001363X

[40] Vervaat, W. (1979). On a stochastic difference equation and a representation of nonnegative infinitely divisible random variables. Adv. in Appl. Probab. 11 750-783. MR0544194 https://doi.org/10.2307/ 1426858

[41] Yin, G.G. and Zhu, C. (2010). Hybrid Switching Diffusions: Properties and Applications. Stochastic Modelling and Applied Probability 63. New York: Springer. MR2559912 https://doi.org/10.1007/ 978-1-4419-1105-6 
[42] Zhang, Z., Tong, J. and Hu, L. (2016). Long-term behavior of stochastic interest rate models with Markov switching. Insurance Math. Econom. 70 320-326. MR3543055 https://doi.org/10.1016/j. insmatheco.2016.06.017

[43] Zhang, Z. and Wang, W. (2017). The stationary distribution of Ornstein-Uhlenbeck process with a two-state Markov switching. Comm. Statist. Simulation Comput. 46 4783-4794. MR3672577 https://doi.org/10.1080/03610918.2015.1132321

Received April 2019 and revised October 2019 